\title{
Balancing expressiveness and inexpressiveness in view design
}

\section{MICHAEL BENEDIKT AND PIERRE BOURHIS AND LOUIS JACHIET AND EFTHYMIA TSAMOURA}

We study the design of data publishing mechanisms that allow a collection of autonomous distributed data sources to collaborate to support queries. A common mechanism for data publishing is via views: functions that expose derived data to users, usually specified as declarative queries. Our autonomy assumption is that the views must be on individual sources, but with the intention of supporting integrated queries. In deciding what data to expose to users, two considerations must be balanced. The views must be sufficiently expressive to support queries that users want to ask - the utility of the publishing mechanism. But there may also be some expressiveness restrictions. Here we consider two restrictions, a minimal information requirement, saying that the views should reveal as little as possible while supporting the utility query, and a non-disclosure requirement, formalizing the need to prevent external users from computing information that data owners do not want revealed. We investigate the problem of designing views that satisfy both expressiveness and inexpressiveness requirements, for views in a restricted declarative language (conjunctive queries), and for arbitrary views.

\section{ACM Reference Format:}

Michael Benedikt and Pierre Bourhis and Louis Jachiet and Efthymia Tsamoura. 2019. Balancing expressiveness and inexpressiveness in view design. ACM Trans. Datab. Syst. 1, 1, Article 1 (January 2019), 45 pages. https://doi.org/10.1145/3365834

\section{INTRODUCTION}

The value of data is increased when data owners make their data available through publicly-accessible interfaces. The value is magnified even further when multiple data owners publish information from related datasets; this allows users to answer queries that require linking information across data sources.

But the benefits of data publishing come with a corresponding risk of revealing too much. For example, there may be information that a data owner wishes to protect, and a user may be able to infer this information either from the published data in isolation, or from the data published by all parties as a whole. There is thus a need to provide data publishing mechanisms that are simultaneously expressive enough to be useful they enable users to answer appropriate queries - while satisfying some expressiveness restriction.

Author's address: Michael Benedikt and Pierre Bourhis and Louis Jachiet and Efthymia Tsamoura.

Permission to make digital or hard copies of all or part of this work for personal or classroom use is granted without fee provided that copies are not made or distributed for profit or commercial advantage and that copies bear this notice and the full citation on the first page. Copyrights for components of this work owned by others than ACM must be honored. Abstracting with credit is permitted. To copy otherwise, or republish, to post on servers or to redistribute to lists, requires prior specific permission and/or a fee. Request permissions from permissions@acm.org.

(C) 2019 Association for Computing Machinery.

0362-5915/2019/1-ART1 \$15.00

https://doi.org/10.1145/3365834

ACM Transactions on Database Systems, Vol. 1, No. 1, Article 1. Publication date: January 2019. 
In data publishing much of the focus has been on disclosure via the familiar mechanism of views - declarative queries whose output is made available to users as a table. In this context, the competing requirements on a publishing mechanism have been primarily studied in isolation. And the focus has been on analysis of a given set of views: we have in front of us some view definitions, and we want to see what properties they satisfy. This is the case in particular for the recent works [7-9]. There is extensive work on analysis of the utility of a set of views: namely given a query, can it be answered using the views, see, e.g. [12, 21]. There has also been research concerning analysis of whether a given set of views obeys some expressiveness restriction. The negation of answerability is clearly too weak a restriction, since it just guarantees that on some instance the query answer cannot be computed from the view images. A relevant querybased notion of expressiveness restriction is data-independent privacy: given the views and a set of "secret" queries, check that the secret query answers cannot be computed from the views on any source data. Variants of data-independent privacy have been studied in [7-9, 27]. Expressiveness restrictions with a similar flavor have also been studied in the context of ontologies [11]. But the question of whether there are expressiveness restrictions for views that do not require the specification of a particular set of secrets, as well as the question of how one obtains views that satisfy both expressiveness and inexpressiveness requirements, has not been considered in the context of traditional queries and views, to the best of our knowledge.

A larger body of work comes from privacy research, considering the design of mechanisms achieving a mix of expressiveness ("utility") and inexpressiveness ("privacy") goals. But the focus is on probabilistic transformations or, more generally, probabilistic protocols (see e.g. $[13,16,17])$. The guarantees are probabilistic, sometimes alternatively or additionally with computational restrictions on an external party. Recent efforts [24] have considered a family of mechanisms that look at database queries, with the utility of a mechanism defined (as in our case) using the notion of query determinacy. But randomness still plays a central role in the mechanism and in the definition of privacy.

In contrast, we consider the question of designing views that use traditional database queries, with no randomization, so that conflicting requirements of expressiveness and inexpressiveness are satisfied. Both our expressiveness and inexpressiveness requirements will be given in terms of exact information-theoretic criteria: they will be defined in terms of what queries can be answered exactly (as opposed to probabilistically) by a party with unlimited computation power. Due to both the difference in the mechanisms we consider and the requirements we impose, our contribution has a very different flavor from prior lines of work. It also differs from work on secure querying over distributed data [6]. There, the goal is to support ad hoc querying of traditional database queries in the presence of privacy restrictions; but they allow the use of encryption as a query language primitive.

Example 1.1. A health study hosted by a government agency holds information about certain treatments, with an abstraction of the data being a database with schema Treatment(pid, tinfo, tdate), where pid is a national insurance number.

Demographic information about patients is stored by another agency, in a table Patient(pid, age, address). The agencies are completely autonomous, perhaps even in 
distinct administrative regions. But they want to co-operate to support certain queries over the data that legitimate researchers might wish; for example about the relationships between treatment and age:

$Q($ tinfo, age $)=\exists$ pid, address, tdate.Treatment $($ pid, tinfo, tdate $) \wedge$ Patient $($ pid, age, address $)$

Of course, the parties could agree to an encryption scheme on the patient identifiers, and then expose encrypted versions of their local data. But this would require both strong co-operation of the parties, and the use of views beyond traditional database queries.

But assuming that the parties are restricted to using traditional queries, what is the most restrictive thing that they can do while supporting the ability to answer $Q$ ? Intuitively, the most restrictive views would correspond to one party revealing the projection of the Treatment table on pid and tinfo, and the other revealing the projection of the Patient table on pid and age.

If this intuition is correct, it would imply that nothing the parties can do with views based on traditional queries can avoid revealing which patients had particular treatments. That is, they have no choice but to allow an external party to learn the answer to query

$$
p=\exists \text { tdate.Treatment(pid, tinfo, tdate) }
$$

Our results will validate this obvious answer - in this example, the two projections of the Patient table described above - are the CQ views that reveal minimal information, and one cannot support the disclosure of the query $Q$ that is joining on attribute pid, while protecting the information concerning the exact pids that are joined. Further we will show that even using encryption - indeed, even using any deterministic function the parties cannot reveal less information while supporting exact answering of the query $Q$.

Example 1.2. Consider a scenario with one source storing a geography-based linking relationship geolink $(x, y)$ between individuals $x$ and $y$, and another storing a social media linking relationship soclink $(x, y)$ between individuals $x$ and $y$. A third source provides information about individual income, which we abstract into a relation Highlncome $(x)$ identifying individuals $x$ with high income.

Consider a query $Q$ asking if there are high-income individuals who are linked geographically and via social media:

$$
Q=\exists x, y \cdot \operatorname{geolink}(x, y) \wedge \operatorname{soclink}(x, y) \wedge \operatorname{Highlncome}(x) \wedge \operatorname{Highlncome}(y)
$$

One can ask what is the minimal information we can reveal in views from the three sources in order to allow the query $Q$ to be answered. In this example, intuitively, it appears difficult to answer the query without revealing all information from the sources. We will see that our results enable us to formalize the notion of minimal information and validate this intuition.

Our goal here is to look at the problem of designing independent views over multiple relational data sources that satisfy both expressiveness and inexpressiveness requirements. Our expressiveness requirement (or usefulness) will be phrased in terms of the ability to answer, using only the information exposed by the views, a relational query - the "utility query" that the parties want to support - where answering is the traditional deterministic notion used in database theory and knowledge representation. 
For expressiveness limitations we will consider two different criteria: the first one asks to find useful views that minimizes the information within the useful views based on a particular class of queries.

Example 1.3. Recall the query $\exists$ pid, address, tdate.Treatment(pid, tinfo, tdate) $\wedge$ Patient(pid, age, address) from Example 1.1, and the views that return the projection of the Treatment table on pid and tinfo in one source, and the projection of the Patient table on pid and age in the other source. These views will be considered useful for the query $Q$, since they are sufficient to answer the query. We will also show that they reveal the minimal information among all useful views.

In Example 1.2, the views that reveal everything from each source - the "identity views" - are clearly sufficient to answer the query $Q$. An external party can just take the view images, which are just the sources themselves, and simply "join them" just as in the definition of $Q$, to answer the queries. Thus, the identity views are "useful for $Q$ " according to our definitions. Conversely, it will follow from our results that, in this example, the identity views actually reveal the minimal information among those which support the answering of $Q$. That is, any useful set of views for $Q$ must reveal the entire content of each data source.

We can also consider a second expressiveness limitation specified by non-disclosure of a set of secret queries.

Example 1.4. We return again to Example 1.2. Suppose that we are tasked with finding views that are useful for $Q$ but where our inexpressiveness condition is to avoid disclosing whether there are high-income individuals who are linked via a chain of three social media links.

In that example we have already conjectured that any views useful for the utility query $Q$ must disclose the entire content of each source, and thus in particular they must disclose the content of the relations soclink and Highlncome. Thus it is intuitively clear that any useful views must disclose the secret.

In comparison, suppose that in Example 1.1 we want to keep secret the set of pairs of individuals having the same address. Here the views that we proposed project out the address information so, intuitively, it is possible to keep this information private.

In contrast to these two examples, we will show that in some cases the parties can obtain combinations of expressiveness and inexpressiveness requirements by using counter-intuitive view combinations. If the reader who wants to get an idea of how complicated such views may need to be, they can do so by looking ahead a bit in this paper. In Example 1.5, we will see that to achieve a balance we may require disjunction in the views, even though our secret query and utility query do not have disjunction. In Example 4.14, the optimal views will require either unsafe disjunction (different free variables in the different disjuncts) or the use of negation. And in the proof of Theorem 6.10 in Section 6 we present a scenario in which one needs to go beyond any standard relational query language to satisfy both an expressiveness and an inexpressiveness restriction.

Our contributions include formalizing these notions, characterizing when minimal information views exist and what form they take, and determining when views exist that satisfy utility and expressiveness limitations. We look at these problems both for views 
given in the standard view language of conjunctive queries, and for arbitrary views. We also consider the impact of background knowledge, in the form of database integrity constraints, on these problems.

Example 1.5. In Example 1.2 suppose background knowledge tells us that the geographic linking relationship is known to be symmetric. We formalize this as a background theory consisting of the following integrity constraint:

$$
\forall x, y \cdot \operatorname{geolink}(x, y) \leftrightarrow \operatorname{geolink}(y, x)
$$

It is now possible to support the utility query while revealing less information. We can change the view on the soclink source to reveal

$$
\operatorname{soclink}(x, y) \vee \operatorname{soclink}(y, x)
$$

It is easy to see that an external party can still answer $Q$ simply by joining the views. Just as clearly, less information is being revealed by this view, since an external party with an access to this views will not be able to distinguish the direction of a social link.

Of course, the reader will observe that we used disjunction in the view above: it is not a conjunctive query. We can show that the "obvious views" from Example 1.3 are still minimally informative within the set of conjunctive query views, even in the presence of this integrity constraint. Thus, we see that - at least in this example - the presence of integrity constraints impacts whether a set of views is minimally informative within the set of all views, but not within the set of CQ views.

Let us now consider the impact of this integrity constraint in terms of diclosure of secrets. An external observer can still learn whether there are high earners that are connected by three social links. They cannot learn this on all databases, since they may be unsure about the direction of a social link. But there are some valid instances where an external party with the access to the views will be able to detect this; this is true in an instance with a high-earner having a social link to themselves.

In fact, we will see that in this example the set of Boolean CQs that are disclosed is not impacted by the presence of these constraints. We therefore see a distinction between distributed views with minimal information and a narrower definition of minimality in terms of the Boolean CQs that are revealed.

Contributions. We will not be able to present a full picture of the view design problem in this work. We will deal only with the case of utility and secret queries given as conjunctive queries, the analogs of SQL basic SELECTs, and we will study only a restricted class of source integrity constraints. But we believe our results suffice to show that our formulation captures an important trade-off in schema design, and that query language expressiveness issues come to the fore. In technical terms, we make the following contributions:

- We formalize the idea of balancing expressiveness and inexpressiveness in distributed views, via the notions of useful distributed views as well as "minimal information" requirements among these views.

- We show that there are useful views with minimal information among the set of CQ views, and also among the set of all views, but that these may differ.

- In contrast to the above, we show that to obtain useful views with minimal information we do not need to go that far beyond CQ views: it suffices to use views defined either using unsafe disjunction of CQ views, or in relational algebra. 
- We show that the above results extend to the presence of background knowledge that is local to each source.

- We examine the impact of background knowledge that relates multiple sources, looking at the simplest kind of relationship, the replication of a relation across sources. We show that we may no longer have useful views with minimal information, but we can get useful views that are minimal in terms of the secrets they disclose. In the process we show that replication can be exploited to allow a view designer to reveal certain queries while not disclosing others.

A diverse set of techniques are applied to study these problems. For investigating the use of CQ views to satisfy both expressiveness and inexpressiveness restrictions, we employ an analysis of the chase proofs that witness determinacy. For studying the use of arbitrary views, both in the absence of background knowledge and in the presence of only local background knowledge, we use a Myhill-Nerode style characterization of when two local sources are interchangeable in terms of their impact on a utility CQ, and then we relate this characterization to certain partial symmetries of the utility queries ("shuffles"). Our analysis of the impact of replication constraints relies on a product construction, which is the key to allowing us to generate useful views that disclose the minimal number of secrets.

Organization. Section 2 gives database and logic preliminaries, and then goes on to formalize our expressiveness and inexpressiveness requirements. Section 3 deals with the variant of the problem where the only views considered are conjunctive query views, while Section 4 shows how the situation changes when arbitrary views can be utilized. Section 5 examines how the situation changes when background knowledge local to a source is present; this is the case of the integrity constraints in Example 1.5. Section 6 consider background knowledge that can connect relations across distinct sources. We close with a discussion of how to interpret the results, future directions, and open questions in Section 7.

\section{PRELIMINARIES}

\subsection{Basic definitions}

The bulk of this subsection reviews standard definitions from databases and knowledge representation. But it includes two notions, DCQs and distributed schemas, that are less standard.

Databases and queries. A schema consists of a finite set of relation names, each with an associated arity (a non-negative number). An instance of a schema is an assignment of each relation name $R$ in the schema of arity $n$ to a collection (finite or infinite) of $n$-tuples of elements. We say that the collection is the interpretation of $R$ in the instance. Given an instance $\mathcal{I}$ and a relation name $R$, let $\mathcal{I}(R)$ be the $n$-tuples assigned to $R$ in $\mathcal{I}$. The active domain of an instance $\mathcal{I}$, denoted adom $(\mathcal{I})$, is the set of elements occurring in some tuple of some $\mathcal{I}(R)$. A fact over an instance $\mathcal{I}$ consists of a relation name $R$ of arity $n$ and an $n$-tuple $\mathbf{t}$ of values $t_{1} \ldots t_{n}$ from the active domain of $\mathcal{I}$. We write such a fact as $R(\mathbf{t})$, and also say that it is a fact over $R$ or an $R$-fact. An instance $\mathcal{I}$ is determined by the set of facts over $\mathcal{I}$, so it equivalently be thought of as a set of facts.

An $n$-ary query is a function from instances of a fixed schema $\mathcal{S}$ to some set. We refer to $\mathcal{S}$ as the input schema of the query. Fix once and for all a set of variables $x_{1} \ldots$ and constants $c_{1} \ldots$ An atom over a schema is an expression $R\left(u_{1} \ldots u_{n}\right)$ where $R$ is an 
$n$-ary relation name over the schema and each $u_{i}$ is either a variable of a constant. An atom $R\left(u_{1} \ldots u_{n}\right)$ is an atom over $R$, and similarly we talk of "facts over $R$ ". A position is a pair $(R, i)$ consisting of a relation name $R$ of some arity $n$ and a number $i$ between 1 and $n$. A position thus represents an argument of a fact or atom over $R$. We will also talk about a position of relation name $R$, meaning a pair $(R, i)$ as above.

A Conjunctive Query (CQ) over a schema $\mathcal{S}$ is a formula of the form $\exists \mathrm{y} . \wedge_{i} A_{i}$ where $A_{i}$ are atoms over the schema. We will often omit "over schema" when it is clear from context or unimportant, and just talk about a CQ. A Boolean CQ (BCQ) is a CQ with no free variables. Following the notation used in several places in the literature (e.g. [2]) we define a Union of CQs (UCQ) to be a disjunction of CQs satisfying the safety condition where the free variables of each disjunct are the same. Disjunctions of CQs where the safety condition is dropped will play a key role in this paper. The terminology for such queries is less standardized, but we refer to them as Disjunctions of CQs (DCQs). UCQs can be extended to relational algebra, the standard algebraic presentation of first-order relational queries: queries are build up from symbols for each relation name by union, difference, selection, and product [1].

For a logical formula $\rho$ with free variables $x_{1}, \ldots, x_{n}$ and an instance $\mathcal{I}$, a variable binding $\sigma$ for $\rho$ in $\mathcal{I}$ is a mapping taking each $x_{i}$ to an element of adom $(\mathcal{I})$. We can apply $\sigma$ to $\rho$ to get a new formula $\sigma(\rho)$ where each $x$ is replaced by $\sigma(x)$. Assuming an ordering of the free variables as $x_{1}, \ldots, x_{n}$ we may identify a variable binding with a $k$-tuple t. In particular, when a certain ordering is assumed we will write $\rho(\mathbf{t})$ to mean that $t_{i}$ is substituted for $x_{i}$ in $\rho$.

A homomorphism between instances $\mathcal{I}_{1}$ and $\mathcal{I}_{2}$ is a function $f$ from $\operatorname{adom}\left(\mathcal{I}_{1}\right)$ to $\operatorname{adom}\left(\mathcal{I}_{2}\right)$ such that for all relation names $R$ and values $c_{1} \ldots c_{m}$ in $\operatorname{adom}\left(\mathcal{I}_{1}\right)$, $R\left(c_{1}, \ldots, c_{m}\right) \in \mathcal{I}_{1}$ implies $R\left(f\left(c_{1}\right), \ldots, f\left(c_{m}\right)\right) \in \mathcal{I}_{2}$.

The canonical database of a CQ $Q$, denoted canondb(Q), is the instance whose facts are the atoms of $Q$, where each variable $v$ corresponds to an element $c_{v}$. The notion of homomorphism from a CQ $Q$ to an instance $\mathcal{I}$ is just a homomorphism from canondb $(Q)$ to $\mathcal{I}$. A homomorphism from a CQ $Q$ to a CQ $Q^{\prime}$ is just a homomorphism between their canonical databases, where we additionally require that the mapping be the identity on any free variables or constants. The output of a CQ $Q$ on an instance $\mathcal{I}$, denoted $Q(\mathcal{I})$ consists of the restrictions to free variables of $Q$ of the homomorphisms of $Q$ to $\mathcal{I}$. The output of a UCQ is defined similarly. We can choose an ordering of the free variables of $Q$, and can then say that the output of $Q$ on $\mathcal{I}$ consists of $n$-tuples. We write $\mathcal{I}, \mathbf{t} \mid=Q$ for an $n$-tuple $\mathbf{t}$, if $\mathbf{t} \in Q(\mathcal{I})$. We analogously define $\mathcal{I}, \sigma \mid=Q$ for a variable binding $\sigma$, and in this case we also say $\mathcal{I}, \sigma$ satisfies $Q$. A CQ with $n$ free variables thus defines an $n$-ary query, and similarly for a UCQ, and more generally any logical formula with $n$ free variables. We sometimes refer to a homomorphism of a BCQ into an instance as a match.

A CQ $Q_{0}$ is a subquery of a CQ $Q$ if the atoms of $Q_{0}$ are a subset of the atoms of $Q$ and a variable of $Q_{0}$ is free in $Q_{0}$ if and only if it is free in $Q$. A strict subquery of $Q$ is a subquery of $Q$ that is not $Q$ itself. A CQ $Q$ is minimal if there is no homomorphism from $Q$ to a strict subquery of $Q$.

A view over a schema $\mathcal{S}$ consists of an $n$-ary relation name $V$ and a corresponding $n$-ary query $Q_{V}$ over relation names from $\mathcal{S}$. Given a collection of views $\mathcal{V}$ and an instance $\mathcal{I}$, the view-image of $\mathcal{I}$, denoted $\mathcal{V}(\mathcal{I})$ is the instance that interprets each 
$\vee \in \mathcal{V}$ by $Q_{\vee}(\mathcal{I})$. We thus talk about $C Q$ views, $U C Q$ views, etc: views defined by formulas within a class.

Distributed data and views. A distributed schema (d-schema) $\mathcal{S}$ consists of a finite set of sources Srcs, with each source s associated with a local schema $\mathcal{S}^{\text {s. We assume }}$ that the local schemas are pairwise disjoint. In Example 1.1, our distributed schema consisted of two sources, one containing Treatment and the other containing Patient. In Example 1.2, our distributed schema consisted of three sources: one containing the list of high-income individuals, one containing the geographical links and one containing social media relationships. We often identify a d-schema with the schema formed by unioning the local schemas. A distributed instance (d-instance) is an instance of the distributed schema, where here, in line with the convention above, we mean the union of the local schemas. For a source $s$, an $s$-instance is an instance of the local schema $\mathcal{S}^{\text {s }}$. Given a d-instance $\mathcal{D}$, we denote by $\mathcal{D}^{\mathrm{s}}$ the restriction of $\mathcal{D}$ to relation names in s. If d-schema $\mathcal{S}$ is parititioned into d-schemas $\mathcal{S}_{1}$ and $\mathcal{S}_{2}$, and we have d-instances $\mathcal{I}_{1}$ for $\mathcal{S}_{1}$ and $\mathcal{I}_{2}$ for $\mathcal{S}_{2}$, then we use $\left(\mathcal{I}_{1}, \mathcal{I}_{2}\right)$ to denote the union of $\mathcal{I}_{1}$ and $\mathcal{I}_{2}$, which is an instance of $\mathcal{S}$. A query over a d-schema is simply a query over the schema formed by taking the union over of relations in all local schemas.

For a given d-schema a distributed view (d-view) $\mathcal{V}$ is an assignment to each source s of a finite set $\mathcal{V}^{\mathrm{s}}$ of views over its local schema. Note that here is our "autonomy" assumption on the instances: we are free to design views on each local source, but the queries defining each view cannot span multiple sources. A CQ-based d-view is a $\mathrm{d}$-view where the set of views on each source is composed of CQs. We can similarly talk about relational algebra-based d-views, etc.

Tuple-generating dependencies and entailment under constraints. We want to consider the impact on disclosure problems of background knowledge. In databases, background knowledge is often modeled using integrity constraints, logical sentences that are assumed to hold on sources. One common class of integrity constraints are Tuple Generating Dependencies (TGDs), which we now review. These are logical sentences of the form $\forall \mathbf{x} . \lambda \rightarrow \exists \mathbf{y} . \rho$, where $\lambda$ and $\rho$ are conjunctions of relational atoms. The notion of a formula $\rho$ holding in an instance $\mathcal{I}$ and a variable binding $\sigma$ (or $\mathcal{I}, \sigma$ satisfying $\rho$, written $\mathcal{I} \mid=\rho$ ) is the standard one in first-order logic. It is consistent with the notion we gave for CQs above. A trigger for $\tau$ in $\mathcal{I}$ is a homomorphism $h$ of $\lambda(\mathbf{x})$ into $\mathcal{I}$. Moreover, a trigger $h$ for $\tau$ is active if no extension of $h$ to a homomorphism of $\rho(\mathbf{x}, \mathbf{y})$ into $\mathcal{I}$ exists. Note that a dependency $\tau$ is satisfied in $\mathcal{I}$ if there is no active trigger for $\tau$ in $\mathcal{I}$.

Let $\Sigma$ be a set of TGDs, $\mathcal{I}$ be a finite instance, and $Q$ be a BCQ. We write $\mathcal{I} \wedge \Sigma \mid=Q$ to mean that every instance containing $\mathcal{I}$ and satisfying $\Sigma$ also satisfies $Q$. For two BCQs $Q$ and $Q^{\prime}$, we similarly write $Q \wedge \Sigma \mid=Q^{\prime}$ to mean that every instance satisfying $Q$ and $\Sigma$ satisfies $Q^{\prime}$. We also say that $Q$ entails $Q^{\prime}$ under $\Sigma$. The terminology extends to CQs by talking about every instance and variable binding. If $\Sigma$ is absent we just say $Q$ entails $Q^{\prime}$. Entailment between CQs is easily seen to be equivalent to existence of a homomorphism from $Q^{\prime}$ to $Q$. Given a set of TGDs $\Sigma$ and another TGD $\sigma_{0}$, we similarly write $\Sigma \mid=\sigma_{0}$ or $\sum$ entails $\sigma_{0}$ to mean that any instance satisfying $\sum$ also satisfies $\sigma_{0}$. The terminology extends in the obvious way to other kinds of logical sentences that have a semantics on instances. 
The chase. The results in Section 3 will make use of the characterization of logical entailment between CQs in the presence of TGDs in terms of the chase procedure $[18,25]$ which we review here. The chase modifies an instance by a sequence of chase steps until all dependencies are satisfied. Let $\mathcal{I}$ be an instance, and consider a TGD $\tau=\forall \mathbf{x} . \lambda \rightarrow \exists \mathbf{y} . \rho$. Let $h$ be a trigger for $\tau$ in $\mathcal{I}$. Performing a chase step for $\tau$ and $h$ to $I$ extends $\mathcal{I}$ with each facts of the conjunction $h^{\prime}(\rho(\mathbf{x}, \mathbf{y}))$, where $h^{\prime}$ is a substitution such that $h^{\prime}\left(x_{i}\right)=h\left(x_{i}\right)$ for each variable $x_{i} \in \mathbf{x}$, and $h^{\prime}\left(y_{j}\right)$, for each $y_{j} \in \mathbf{y}$, is a fresh labeled null that does not occur in $\mathcal{I}$.

For $\Sigma$ a set of TGDs and $\mathcal{I}$ an instance a chase sequence for $\Sigma$ and $\mathcal{I}$ is a (possibly infinite) sequence $\mathcal{I}_{0}, \mathcal{I}_{1}, \ldots$ such that $\mathcal{I}=\mathcal{I}_{0}$ and, for each $\mathcal{I}_{i}$ with $i>0$ is obtained from $\mathcal{I}_{i-1}$ by applying a successful chase step to a dependency $\tau \in \Sigma$ and an active trigger $h$ for $\tau$ in $\mathcal{I}_{i-1}$. The sequence must be fair: for each $\tau \in \Sigma$, each $i \geq 0$, and each active trigger $h$ for $\tau$ in $\mathcal{I}_{i}$, some $j>i$ must exist such that $h$ is not an active trigger for $\tau$ in $\mathcal{I}_{j}$ The result of a chase sequence is the (possibly infinite) instance $\mathcal{I}_{\infty}=\bigcup_{i \geq 0} \mathcal{I}_{i}$. We use $\operatorname{Chase}_{\Sigma}(\mathcal{I})$ to denote the result of any chase sequence for $\Sigma$ on $\mathcal{I}$.

A finite chase sequence is terminating. A set of dependencies $\Sigma$ has terminating chase if, for each finite, instance $\mathcal{I}$, each chase sequence for $\Sigma$ and $\mathcal{I}$ is terminating. For such $\Sigma$, the chase provides an effective approach to testing if $\mathcal{I} \wedge \Sigma \mid=Q$ : we compute (any) chase $\mathcal{I}_{\infty}$ for $\Sigma$ and $\mathcal{I}$ and check if $Q$ holds [18]. We can similarly test if $Q \wedge \Sigma \mid=Q^{\prime}$ by chasing canondb $(Q)$ with $\Sigma$ and then checking $Q^{\prime}$. Checking if a set of dependencies $\Sigma$ has terminating chase is undecidable [15]. Weak acyclicity [18] was the first sufficient polynomial-time condition for checking if $\Sigma$ has terminating chase. Stronger sufficient (not necessarily polynomial-time) conditions have been proposed subsequently $[14,29]$.

\subsection{Problem formalization}

We now give the key definitions in the paper, capturing our expressiveness requirements ("useful") and expressiveness limitations ("minimally informative" and "nondisclosing"). Our expressiveness requirement is via the concept of determinacy [28], formalizing the idea that on any instance there is sufficient information in the views to recapture the query.

Definition 2.1. Two d-instances $\mathcal{D}$ and $\mathcal{D}^{\prime}$ are indistinguishable by a d-view $\mathcal{V}$ (or just $\mathcal{V}$-indistinguishable) if $V(\mathcal{D})=V\left(\mathcal{D}^{\prime}\right)$ for each view $V \in \mathcal{V}$.

Since each view $V \in \mathcal{V}^{\mathrm{s}}$ is defined only using relation names occurring in $\mathcal{V}^{\mathrm{s}}$, we can equivalently say that $\mathcal{D}_{1}$ and $\mathcal{D}_{2}$ are $\mathcal{V}$-indistinguishable if $V\left(\mathcal{D}_{1}^{s}\right)=V\left(\mathcal{D}_{2}^{s}\right)$ for each $V \in \mathcal{V}^{\text {s }}$ for each source s.

Definition 2.2. A d-view $\mathcal{V}$ determines a query $Q$ at a d-instance $\mathcal{D}$ if $Q\left(\mathcal{D}^{\prime}\right)=Q(\mathcal{D})$ for each $\mathcal{D}^{\prime}$ that is $\mathcal{V}$-indistinguishable from $\mathcal{D}$.

The d-view $\mathcal{V}$ is useful for $Q$ if $\mathcal{V}$ determines $Q$ on every d-instance (for short, just “ $V$ determines $Q ")$.

Usefulness for a given query $Q$ will be our expressiveness requirement on d-views. Our first inexpressiveness requirement captures the idea that we want to reveal as little as possible: 
Definition 2.3 (Minimally informative useful views). Given a class of views $C$ and a query $Q$, we say that a d-view $\mathcal{V}$ is a minimally informative useful $d$-view for $Q$ within $C$ if $\mathcal{V}$ is useful for $Q$ and, for any other d-view $\mathcal{V}^{\prime}$ useful for $Q$ based on views in $C$, $\mathcal{V}^{\prime}$ determines the view definition of each view in $\mathcal{V}$.

We look at another inexpressiveness requirement that requires an external party to not learn about another query.

Definition 2.4 (Non-disclosure). A non-disclosure function specifies, for each query $p$, a set of d-views $\mathcal{V}$ that are said to disclose $p$. We require such a function $F$ to be determinacy-compatible: if $\mathcal{V}_{2}$ discloses $p$ according to $F$ and $\mathcal{V}_{1}$ determines each view in $\mathcal{V}_{2}$, then $\mathcal{V}_{1}$ also discloses $p$ according to $F$. If $\mathcal{V}$ does not disclose $p$, then we say that $\mathcal{V}$ is non-disclosing for $p$ (relative to the given non-disclosure function).

When we can find minimally informative useful d-views, this tells us something about non-disclosure, since it is easy to see that if we are looking to design views that are useful and non-disclosing, it suffices to consider minimally informative views, assuming they exist:

PROPOSITION 2.5. Suppose $\mathcal{V}$ is a minimally informative useful d-view for $Q$ within $C$, and there is a d-view based on views in $C$ that is useful for $Q$ and non-disclosing for $p$ according to non-disclosure function $F$. Then $\mathcal{V}$ is useful for $Q$ and non-disclosing for $p$ according to $F$.

There are many non-disclosure functions that are determinacy-compatible. But in our examples, our complexity results, and in Section 6, we will focus on a specific non-disclosure function, whose intuition is that an external party "never infers any answers".

Definition 2.6 (UN Non-disclosure). A d-view $\mathcal{V}$ is universal non-inference nondisclosing (UN non-disclosing) for a CQ $p$ if for each instance $\mathcal{I}$ and each tuple $\mathbf{t}$ with $\mathcal{I}, \mathbf{t}=p, \mathcal{V}$ does not determine $p(\mathbf{t})$ at $\mathcal{I}$. Otherwise, the d-view $\mathcal{V}$ is said to be $U N$ disclosing for $p$.

This non-disclosure function is clearly determinacy-compatible, so Proposition 2.5 will apply to it. Thus we will be able to utilize UN non-disclosure as a means of showing that certain d-views are not minimally informative.

Variations: background knowledge, and finite instances. All of these definitions can be additionally parameterized by background knowledge $\Sigma$, consisting of integrity constraints in some logic. We will refer to an arbitrary finite set of such sentences as a theory or a set of integrity constraints. Given d-instance $\mathcal{D}$ satisfying $\Sigma$ and a d-view $\mathcal{V}$, $\mathcal{V}$ determines a query $Q$ over the d-schema at $\mathcal{D}$ relative to $\Sigma$ if: for every $\mathcal{D}^{\prime}$ satisfying $\Sigma$ that is $\mathcal{V}$-indistinguishable from $\mathcal{D}, Q\left(\mathcal{D}^{\prime}\right)=Q(\mathcal{D})$. We say that $\mathcal{V}$ is useful for a query $Q$ relative to $\Sigma$ if it determines $Q$ on every d-instance satisfying $\Sigma$. We say $\mathcal{V}$ is UN non-disclosing for query $p$ with respect to $\Sigma$ if $\mathcal{V}$ does not determine $p$ on any $d$-instance satisfying $\Sigma$.

By default, when we say "every instance", we mean all instances, finite or infinite. There are variations of this problem requiring the quantification in both non-disclosure and utility to be over finite instances. The advantage of dealing with the unrestricted variant of the problem is that for views and queries in relational algebra the determinacy 
problem is semi-decidable, and is equivalent to the standard notion of rewritability in relational algebra [28]. In contrast, the finite version is not semi-decidable even for conjunctive queries and views [20]. The use of the unrestricted version will also allow us to make use of the chase construction, which will be convenient in Section 3. However, it will turn out that the main results in the paper do not depend on this design choice, and hold for either version of the definition: see Section 7 for further discussion.

Main problem. We focus on the problem of determining whether minimally informative useful d-views exist for a given query $Q$ and class $C$, and characterizing such views when they do exist. When minimally informative useful d-views do not exist, we consider the problem of obtaining a d-view that is useful for $Q$ and which minimizes the set of secrets $p$ that are UN disclosed for $p$. We refer to $Q$ as the utility query, and to $p$ as the secret query. We investigate this problem both in the presence and the absence of integrity constraints.

Example 2.7. In Example 1.2 our scenario involved the utility query $\exists x, y$.geolink $(x, y) \wedge$ $\operatorname{soclink}(x, y) \wedge \operatorname{HighIncome}(x) \wedge \operatorname{Highlncome}(y)$. Our results will validate the intuition stated there, that the minimally informative useful d-view, in the absence of constraints, is one which simply reveals the entire database! Our results will also allow us to formalize the intuition concerning the impact of the symmetry constraint in Example 1.5: to reveal minimal information, we no longer return the entire instance, but utilize a disjunction on the soclink source. From these observations, we will easily conclude that even in the presence of the symmetry constraint, any view that is useful for this query must reveal the answer to the secret query asking high-income individuals who are linked via a chain of three social media links.

Discussion of utility and non-disclosure definitions. Our formalization of utility for views is information-theoretic and exact: a view is useful if a party with access to the view can compute the exact output of the query (as opposed to the correct output with high probability), without limiting the computation. The generality of this notion will make our negative results stronger. And it turns out the generality will not limit our positive results, since these will be realized by very simple views.

Our formalization of minimally informative views is likewise natural if one seeks an ordering on sets of views measuring the ability to support exact information-theoretic query answering. Our query-based inexpressiveness notion, non-disclosure, gives a way of seeing the impact of minimally informative useful views on protecting information, and it is also based on information-theoretic and exact criteria. We exemplify our general definition of non-disclosure function with UN non-disclosure, which has been studied in prior work under several different names [7-9, 27]. We choose the name "non-disclosing" rather than "private" for all our query-based expressiveness restrictions, since they are clearly very different from more traditional probabilistic privacy guarantees [17].

The reader may have noted that UN non-disclosure only deals with protecting positive information about a secret query $p$. The attacker can still infer that the query is not true on an instance. One can modify the definition in the obvious way to deal with negative information. One still obtains a valid non-disclosure function, and all of our results up until those in Section 6 will still hold for it, other than those referring to complexity. But notice that this enhancement of UN non-disclosure is impossible to achieve in the absence of integrity constraints, or even if there are integrity constraints but only TGDs: 
the empty instance is always a counterexample! This variant thus becomes interesting only when more general integrity constraints are allowed. The analysis of a given set of views for this variant of UN non-disclosure is investigated in [8].

On the one hand the UN non-disclosure guarantee, along with its variant with negative information above, is weak in that $p$ is considered safe for $\mathcal{V}$ (UN non-disclosed) if an attacker can never infer something about $p$ with absolute certainty. Given a distribution on source instances, the information in the views may still increase the likelihood that $p$ holds. On the other hand, UN non-disclosure and its variant above are quite strong in that they must hold on every valid source instance. As with the positive/negative distinction discussed a paragraph above, we can attempt to address both of these issues by strengthening or weakening the definition to include a more fine-grained quantitative analysis. For example we can say that a secret $p$ is not disclosed if, for each number $k$, there are at most a quarter of the instances of size $k$ where an external party can be sure that $p$ is true. This is once again a valid non-disclosure function, and all of of our expressiveness results for such functions will apply to it. However, we do not know how to analyze such complex functions. And even to see whether these quantitative notions of non-disclosure properties hold in particular examples is a challenging problem, outside the scope of this work.

Thus, although we do not by any means claim that UN non-disclosure captures all intuitively desirable properties of privacy, we do feel that it allows us to explore the ability to create views that simultaneously support the strong ability to answer certain queries in data integration and the strong inability to answer other queries. We also make use of it as a tool to understand minimal information.

Restrictions and simplifications. Although the utility and non-disclosure definitions above make sense for any queries, for simplicity, in the remainder of this paper we will assume that $Q$ and $p$ are BCQs without constants. We will thus abuse notation by referring to a BCQ without constants as simply a BCQ. Our results generalize easily in the presence of constants. While we restrict to the case of a single utility query and secret query here, all of our results have easy analogs for a finite set of such queries.

\subsection{Some tools}

Throughout the paper we rely on two basic tools.

Canonical views. Recall that we are looking for views that are useful for answering a CQ $Q$ over a d-schema. The "obvious" set of views to try are those obtained by partitioning the atoms of $Q$ among sources, with the free variables of the views including the free variables of $Q$ and the variables occurring in atoms from different sources.

Given a CQ $Q$ over a d-schema, and a source $s$, we denote by $\operatorname{SVars}(s, Q)$ the variables of $Q$ that appear in an atom from source $s$. We also denote by $\operatorname{SJVars}(s, Q)$ the "sourcejoin variables of $s$ " in $Q$ : the variables in $\operatorname{SVars}(s, Q)$ and that also occur in an atom of another source.

Definition 2.8. The canonical view of $Q$ for source $s, \operatorname{CanView}^{s}(Q)$, has a view definition formed by conjoining all $s$-source atoms in $Q$ and then existentially quantifying all bound variables of $Q$ in $\operatorname{SVars}(s, Q) \backslash \operatorname{SJVars}(s, Q)$. The canonical d-view of $Q$ is formed by taking the canonical view for each source. 
In Example 1.1, the canonical d-view is what we referred to as "the obvious view design". It would mean that one source has a view exposing $\exists$ tdate.Treatment(pid, tinfo, tdate) - since pid is a source-join variable while tinfo is a free variable of $Q$. The other source should expose a view revealing $\exists$ address.Patient(pid, age, address), since address is neither a free variable nor shared across sources.

The critical instance. In the definition of UN non-disclosure of a query by a set of views, we required that on any instance of the sources, a user who has access to the views cannot reconstruct the answer to the query $p$. An instance that will be helpful in several examples is the following "most problematic" instance [26].

Definition 2.9. The critical instance of a schema $\mathcal{S}$ is the instance whose active domain consists of a single element $*$ and whose facts are $R(*, \ldots, *)$ for all relation names $R$ in $\mathcal{S}$.

Note that every BCQ over the relevant relation names holds on the critical instance of the source. The critical instance is the hardest instance for UN non-disclosure in the following sense:

THEOREM 2.10. [8, 9] Consider any $C Q$ views $\mathcal{V}$, and any $B C Q p$. If $p$ is determined by $\mathcal{V}$ at some instance of the source schema, then it is determined by $\mathcal{V}$ at the critical instance. The same holds when these definitions are relativized to a theory consisting of TGDs.

Note that this theorem is the only significant result that we take from $[8,9]$, and that it will be used only for understanding examples and in Section 6.

\section{BALANCING EXPRESSIVENESS AND INEXPRESSIVENESS WITH CQ VIEWS}

We now begin our analysis of balancing expressiveness and inexpressiveness. Returning to Example 1.1, recall the intuition that the canonical d-view of $Q$ is the "least informative d-view" that supports the ability to answer $Q$. We would thus expect the canonical d-view to be a minimally information useful d-view as defined in Section 2 . We start by proving such a result, but with two restrictions: the utility query $Q$ must be a minimal $\mathrm{CQ}$, and we only consider views specified by CQs:

THEOREM 3.1. [Minimally informative useful CQ views] For every minimal $B C Q$ $Q$, the canonical d-view of $Q$ is minimally informative within $C Q$-based d-views. That is, if any $C Q$-based $d$-view $\mathcal{V}$ determines a $Q$, then $\mathcal{V}$ determines each canonical view $\operatorname{CanView}^{\mathrm{s}}(Q)$ of $Q$.

We present a proof of this result, making use of the chase construction reviewed in Section 2. We note that this result will also follow from a more general theorem presented in Subsection 5.2, which will rely on techniques presented later concerning arbitrary views. All proofs make use of the following property of minimal CQs, which is easy to verify:

LEMMA 3.2. If $Q$ is a minimal $C Q$, then there is no homomorphism $h$ from $Q$ into itself that maps two different variables occurring in $Q$ to the same variable.

We will begin the chase-based proof by showing that the determinacy of a CQ $Q$ by a set of CQ views $\mathcal{V}$ leads to the existence of a certain homomorphism of $Q$ to itself. 
Let $Q$ be a BCQ and $\mathcal{V}$ be an arbitrary set of CQ-views. We fix a signature for our queries and views, which we refer to as the original signature. From it, we derive a primed signature, containing a relation name $R^{\prime}$ for each $R$ in the original signature. Given a formula $\varphi$ in the original signature, we let $\varphi^{\prime}$ be formed by replacing every relation name $R$ in $\varphi$ by $R^{\prime}$. We use a similar notation for a set of facts $S$ in the original signature. In particular, for a conjunctive query $Q$ in the original signature, $Q^{\prime}$ refers to the conjunctive query obtained by replacing every relation name by its primed counterpart.

Given a view $\mathrm{V}(\mathbf{x})$ defined by conjunctive query $\exists \mathbf{y} . \varphi(\mathbf{x}, \mathbf{y})$, the forward view definition for $\mathrm{V}$ is the TGD:

$$
\varphi(\mathbf{x}, \mathbf{y}) \rightarrow \mathrm{V}(\mathbf{x})
$$

while the inverse view definition for $\mathrm{V}$ is the TGD:

$$
\mathrm{V}(\mathbf{x}) \rightarrow \exists \mathbf{y} \cdot \varphi(\mathbf{x}, \mathbf{y})
$$

For any CQ views $\mathcal{V}$, let $\Sigma_{\mathcal{V}}$ denote the set of TGDs consisting of the forward and inverse view definitions for views in $\mathcal{V}$, as well as the forward and inverse view definitions but for primed copies of the base predicates.

Example 3.3. Suppose we have a set of views $\mathcal{V}$ consisting only of view $V(x)$ given by CQ $\exists y, z \cdot R(x, y) \wedge S(x, z)$. Then $\Sigma_{\mathcal{V}}$ contains the TGDs:

$$
\begin{aligned}
& R(x, y) \wedge S(x, z) \rightarrow V(x) \quad \text { forward view } \\
& R^{\prime}(x, y) \wedge S^{\prime}(x, z) \rightarrow V(x) \quad \text { forward prime view } \\
& V(x) \rightarrow \exists y, z \cdot R(x, y) \wedge S(x, z) \quad \text { backward view } \\
& V(x) \rightarrow \exists y, z \cdot R^{\prime}(x, y) \wedge S^{\prime}(x, z) \quad \text { backward prime view }
\end{aligned}
$$

It is easy to see that, for any CQ views $\mathcal{V}$ and any CQ $Q$, determinacy of $Q$ by $\mathcal{V}$ can be expressed as:

$$
Q \wedge \Sigma_{\mathcal{V}}=Q^{\prime}
$$

This is a containment of CQs under TGDs, and the chase algorithm checks this and is complete for such containments (see Section 2). Intuitively, the chase algorithm produces an infinite instance $\operatorname{Chase}_{\Sigma_{v}}(\operatorname{canondb}(Q))$, and then checks for a match of $Q^{\prime}$. Note that, because it produces an infinite model, the chase algorithm might not terminate. But it always terminates when $Q \wedge \Sigma_{\mathcal{V}}=Q^{\prime}$. We summarize these observations with the following proposition:

PROPOSITION 3.4. For each query $Q$ and set of views $\mathcal{V}$, the following three statements are equivalent:

- $Q \wedge \Sigma_{\mathcal{V}} \mid=Q^{\prime}$

- $\mathcal{V}$ determines $Q$;

- there exists a homomorphism of canondb $\left(Q^{\prime}\right)$ into $\operatorname{Chase}_{\Sigma_{v}}(\operatorname{canondb}(Q))$ that is the identity on values $c_{v}$ where $v$ is a free variable of $Q$.

The result is implicit in [28], and proofs can be found in [10, 19].

We are now ready for the proof of Theorem 3.1:

Proof. Assume that $\mathcal{V}$ determines $Q$ and thus, from Proposition 3.4 there exists a homomorphism $h_{1}$ from canondb $\left(Q^{\prime}\right)$ into Chase $_{\Sigma_{v}}(\operatorname{canondb}(Q))$, or more precisely, 
from canondb $\left(Q^{\prime}\right)$ into the instance consisting of the primed facts of Chase $\Sigma_{\mathcal{V}}(\operatorname{canondb}(Q))$. We can equivalently think of $h_{1}$ as having domain $Q^{\prime}$, and we will use this perspective in the proof.

We now observe that the building of $\operatorname{Chase}_{\Sigma_{v}}(\operatorname{canondb}(Q))$ can be done independently on the different sources. Indeed with our autonomy assumption, we have that $Q \wedge \Sigma_{\mathcal{V}}=\bigwedge_{s} \operatorname{CanView}^{\mathrm{s}}(Q) \wedge \Sigma_{\mathcal{V}^{\mathrm{s}}}$, where we recall that $\mathcal{V}^{\mathrm{s}}$ denotes the views in $\mathcal{V}$ that apply to source s.

The chase process also decomposes by sources:

$$
\operatorname{Chase}_{\Sigma_{V}}(\operatorname{canondb}(Q))=\bigcup_{s} \operatorname{Chase}_{\Sigma \mathcal{V}^{s}}\left(\operatorname{canondb}\left(\operatorname{CanView}^{\mathrm{s}}(Q)\right)\right)
$$

Thus, the active domains of $\mathrm{Chase}_{\Sigma_{\gamma s}}\left(\operatorname{canondb}\left(\operatorname{CanView}^{\mathrm{s}}(Q)\right)\right)$ for the distinct sources $s$ are all disjoint except for the values corresponding to source-join variables. As a consequence, for each value corresponding to a source-join variable $c_{v}, h_{1}\left(c_{v}\right)$ must be a value shared between different sources and thus $h_{1}$ must map source-join variables to values corresponding to source-join variables.

Looking ahead to our goal, we want to show that $\mathcal{V}$ determines each canonical view CanView ${ }^{\mathrm{s}}(Q)$. Fix a source $\mathrm{s}_{0}$. Applying Proposition 3.4 again, we see that our determinacy goal is equivalent to finding a homomorphism of $\operatorname{CanView}^{\mathrm{s}_{0}}\left(Q^{\prime}\right)$ into Chase $_{\Sigma_{v}}\left(\mathrm{CanView}^{\mathrm{s}_{0}}(Q)\right)$, that is the identity on the source-join variables. The restriction of $h_{1}$ to variables in $\operatorname{CanView}^{\mathrm{s}_{0}}(Q)$ is thus almost enough to give us our desired conclusion, except that it may not be the identity on source-join variables. In order to fix this issue we will need to translate between homomorphisms like $h_{1}$ and a homomorphism from $Q$ to itself. This will allow us to make use of the assumption that $Q$ is a minimal CQ.

Let $\mathcal{D}=\operatorname{canondb}\left(Q^{\prime}\right) \cup \operatorname{canondb}(Q) \cup \mathcal{V}($ canondb $(Q))$. Note that the constraints of $\Sigma_{\mathcal{V}}$ hold in $\mathcal{D}$ and thus we can assume $\operatorname{Chase}_{\Sigma_{\mathcal{V}}}(\mathcal{D})=\mathcal{D}$. Observe also that canondb $(Q) \subseteq \mathcal{D}$, and we can extend this to a homomorphism as we chase. This means there is a homomorphism $h_{2}$ from $\operatorname{Chase}_{\Sigma_{v}}(\operatorname{canondb}(Q))$ into Chase $\Sigma_{\Sigma_{V}}(\mathcal{D})$ which is the identity on canond $(Q)$ and, in particular, is the identity on values corresponding to source-join variables. If we look only at the instance consisting of primed atoms we see that $h_{2}$ is a homomorphism from the primed atoms of Chase $\Sigma_{\mathcal{V}}(\operatorname{canondb}(Q))$ to the primed atoms of $\operatorname{Chase}_{\Sigma_{V}}(\mathcal{D})=\mathcal{D}$ and the primed atoms of $\mathcal{D}$ are exactly canondb $\left(Q^{\prime}\right)$. In summary, we have a homomorphism $h_{2}$ from the primed atoms of Chase $_{\Sigma_{V}}(\operatorname{canondb}(Q))$ to canondb $\left(Q^{\prime}\right)$.

We let $h_{Q}=h_{2} \circ h_{1}$, this is a homomorphism from canondb $\left(Q^{\prime}\right)$ to itself. Figure 1 gives a graphical representation of the situation. We now consider two cases.

Case 1: $h_{Q}$ is not injective. By unpriming and using the canonical homomorphism from $Q$ to canondb $(Q)$ and its reverse, we obtain a non-injective homomorphism from $Q$ to itself. But now, by Lemma 3.2, we have a contradiction of minimality.

Case 2: $h_{Q}$ is injective. Since $h_{Q}$ is a total function on a finite domain, it must be bijective. What we need to prove, by Proposition 3.4, is the existence of a homomorphism $h$ from canondb $\left(\operatorname{CanView}^{\mathrm{s}_{0}}\left(Q^{\prime}\right)\right)$ into $\operatorname{Chase}_{\Sigma_{\mathcal{V}_{0}^{\mathrm{s}}}}\left(\operatorname{canondb}\left(\operatorname{CanView}^{\mathrm{s}}(Q)\right)\right)$, where the homomorphism must be the identity on values corresponding to free variables of CanViews $\left(Q^{\prime}\right)$ (i.e. the source-join variables of $Q^{\prime}$ ). 


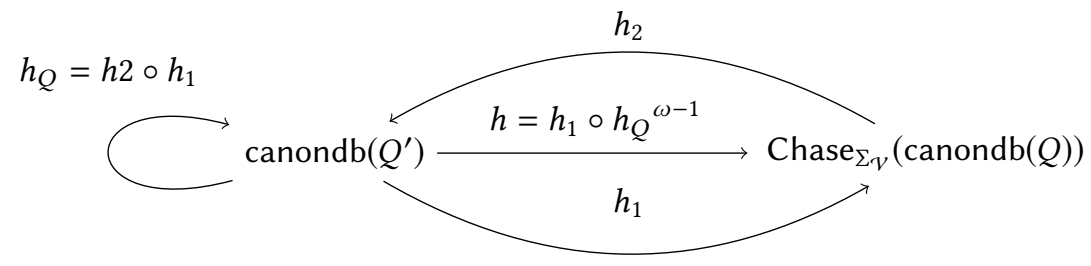

Fig. 1. Representation of the homomorphisms introduced for proof of Proposition 3.4

Since $h_{Q}$ is bijective, for some number $\omega>0, h_{Q}{ }^{\omega}$ is the identity. We then look at $h=h_{1} \circ\left(h_{Q}\right)^{w-1}$. This is a homomorphism from canondb $\left(Q^{\prime}\right)$ to Chase $_{\Sigma_{v}}(\operatorname{canondb}(Q))$ (see Figure 1). We already know that $h_{2} \circ h=h_{Q}{ }^{\omega}$ is the identity but since $h_{2}$ is already the identity on source-join variables, it must be that $h$ also is. Therefore $h$ restricted to $\mathrm{s}_{0}$ is the homomorphism we were looking for.

Consequences. Combining Theorem 3.1 and Proposition 2.5 gives a partial answer to the question of how to obtain useful and non-disclosing views:

COROLLARY 3.5. For any non-disclosure function $F$, and for any BCQs $Q$ and $p$, if there is some CQ-based d-view that is useful for $Q$ and non-disclosing for $p$ according to $F$, then the canonical $d$-view of $Q^{\mathrm{min}}$ is such a $d$-view, where $Q^{\mathrm{min}}$ is any minimal $C Q$ equivalent to $Q$.

If we consider the specific non-disclosure notion, UN non-disclosure, we can infer a complexity bound from combining these results with prior work on the complexity of checking non-disclosure (Theorem 44 of [9]):

COROLLARY 3.6. There is a $\Sigma_{2}^{p}$ algorithm taking as input BCQs $Q$ and $p$ and determining whether there is a CQ-based d-view that is useful for $Q$ and UN nondisclosing for $p$. If $Q$ is assumed minimal, then the problem in CoNP.

We will not investigate the algorithmic consequences more thoroughly, since they are not the focus of the paper. We note that determining whether the canonical views of $Q$ are UN non-disclosing for a query $p$ can be decomposed into UN non-disclosure questions on each source: whether or not the canonical view of $p$ for a given source $s$ is UN disclosed relative to the canonical view of $Q$ for $s$. This reduces the complexity to $\Sigma_{2}^{p}$ in the maximum size of the canonical views for $Q$ and $p$ over all sources, which could be considerably smaller than the size of $Q$ and $p$ itself.

The following example shows that the requirement that $Q$ is minimal (that is, has no redundant conjuncts) in Theorem 3.1 is essential.

Example 3.7. Consider two sources. The first source comprises the relation names $R_{1}$, $R_{2}$ and $R_{3}$, while the second source comprises a single relation name $T$. Consider also the conjunctions of atoms $C_{1}$ and $C_{2}$ defined as:

$$
\begin{aligned}
& C_{1}=R_{1}(x, y) \wedge T(x) \\
& C_{2}=R_{1}\left(x^{\prime}, y^{\prime}\right) \wedge R_{1}\left(y^{\prime}, z^{\prime}\right) \wedge R_{1}\left(z^{\prime}, x^{\prime}\right) \wedge T\left(x^{\prime}\right) \wedge R_{2}\left(y^{\prime}\right) \wedge R_{3}\left(z^{\prime}\right)
\end{aligned}
$$


The conjunction $C_{1}$ states that there is an element in $T$ that is the source of an $R$ edge. The conjunction $C_{2}$ states that there is an $R_{1}$-triangle with one vertex in $T$, a second in $R_{2}$, and a third in $R_{3}$. Consider now the BCQ $Q$ defined as

$$
\exists x, y, x^{\prime}, y^{\prime}, z^{\prime} . C_{1} \wedge C_{2}
$$

Note that the conjunction of atoms $C_{1}$ in $Q$ is redundant. Indeed, the BCQ

$$
Q^{\text {min }}=\exists x^{\prime}, y^{\prime}, z^{\prime} . C_{2}
$$

is equivalent to $Q$.

The canonical view of $Q$ for the $R_{i}$ 's source is:

$$
\exists y, y^{\prime}, z^{\prime} . R_{1}(x, y) \wedge R_{1}\left(x^{\prime}, y^{\prime}\right) \wedge R_{1}\left(y^{\prime}, z^{\prime}\right) \wedge R_{1}\left(z^{\prime}, x^{\prime}\right) \wedge R_{2}\left(y^{\prime}\right) \wedge R_{3}\left(z^{\prime}\right)
$$

But the canonical view of $Q^{\min }$ for this source is

$$
\exists y^{\prime}, z^{\prime} . R_{1}\left(x^{\prime}, y^{\prime}\right) \wedge R_{1}\left(y^{\prime}, z^{\prime}\right) \wedge R_{1}\left(z^{\prime}, x^{\prime}\right) \wedge R_{2}\left(y^{\prime}\right) \wedge R_{3}\left(z^{\prime}\right)
$$

Theorem 3.1 tells us that the canonical d-view of $Q^{\min }$ is a minimally informative useful d-view within the class of CQ views. We claim that the canonical d-view of $Q$ is not minimally informative for this class, and in fact reveals significantly more than the canonical d-view of $Q^{\mathrm{min}}$. Consider the secret query $p=\exists t \cdot R_{1}(t, t)$ stating that there is an $R_{1}$ self-loop. The canonical d-view of $Q^{\mathrm{min}}$ is $\mathrm{UN}$ non-disclosing for $p$. Indeed, given any instance $\mathcal{D}$, consider the instance $\mathcal{D}^{\prime}$ in which the $T$ source is identical to the one in $\mathcal{D}$, but the $R$ source is replaced by one where each node $e$ in the canonical view for $\mathcal{D}$ is in a triangle with distinct elements for $y^{\prime}, z^{\prime}$. Such a $\mathcal{D}^{\prime}$ does not satisfy $p$, and is indistinguishable from $\mathcal{D}$ according to the canonical d-view of $Q^{\min }$.

In contrast, the canonical d-view of $Q$ is UN disclosing for $p$. Consider $\mathcal{D}_{0}$, the critical instance for the source schema (see Section 2). On $\mathcal{D}_{0}$ the returned bindings have $x$ as only $*$, and from this we can infer that the witness elements for $y^{\prime}$ and $z^{\prime}$ can only be $*$, and hence the d-view discloses $p$ with $\mathcal{D}_{0}$ as the witness.

By Proposition 2.5 the canonical d-view of $Q$ cannot be minimally informative within the class of CQ views.

\section{ARBITRARY VIEWS}

In Section 3 we showed that the canonical d-view is a minimally informative useful view within the class of CQ views, assuming that the utility query is minimized as a CQ. We now turn to minimally informative useful d-views, not restricting to views given by CQ view definitions.

Our goal will be to arrive at a generalization of the notion of canonical d-view gives the minimal information over arbitrary useful d-views for a given BCQ $Q$. That is, we want to arrive at an analog of Theorem 3.1 replacing "CQ views" by "arbitrary views" and "canonical view" by a generalization.

\subsection{An equivalence class representation of minimally informative views}

Recall that general views are defined by queries, where a query can be any function on instances. An Equivalence Class Representation of a d-view (ECR) consists of an equivalence relation $\equiv^{\mathrm{s}}$ for each source $s$. An ECR is just another way of looking at a d-view defined by a set of arbitrary functions on instances: given a function $F$, one can 
define an equivalence relation by identifying two local instances when the values of $F$ are the same. Conversely, given an equivalence relation, one can define a function mapping each instance to its equivalence class. A d-instance $\mathcal{D}_{1}$ is indistinguishable from a d-instance $\mathcal{D}_{2}$ by the d-view specified by ECR $\left\langle\equiv^{\mathrm{s}}\right.$ : s $\left.\in \operatorname{Srcs}\right\rangle$ when $\mathcal{D}_{1}^{\mathrm{s}} \equiv^{\mathrm{s}} \mathcal{D}_{2}^{\mathrm{s}}$ holds for each source s. Determinacy of one d-view by another d-view corresponds exactly to the refinement relationship between the corresponding ECRs. We will thus abuse notation by talking about indistinguishability, usefulness, and minimal informativeness of an ECR, referring to the corresponding d-view.

Our first step will be to show that there is an easy-to-define ECR whose corresponding $\mathrm{d}$-view is minimally informative. For a source $\mathrm{s}$, an s-context is an instance for each source other than s. Given an s-context $C$ and an s-instance $\mathcal{I}$, we use $(\mathcal{I}, C)$ to denote the d-instance formed by interpreting the s-relation names as in $\mathcal{I}$ and the others as in C.

We say two s-instances $\mathcal{I}, \mathcal{I}^{\prime}$ are (s, $\left.Q\right)$-equivalent if for any s-context $C$,

$$
(\mathcal{I}, C)\left|=Q \Leftrightarrow\left(\mathcal{I}^{\prime}, C\right)\right|=Q
$$

We say two d-instances $\mathcal{D}$ and $\mathcal{D}^{\prime}$ are globally $Q$-equivalent if for each source $s$, the restrictions of $\mathcal{D}$ and $\mathcal{D}^{\prime}$ over source $s$, are (s, $Q$ )-equivalent.

Global $Q$-equivalence is clearly an ECR. It is also not difficult to see that the corresponding d-view is a minimally informative useful d-view for $Q$ within the class of all views:

PROPOSITION 4.1. The d-view corresponding to global Q-equivalence is a minimally informative useful $d$-view for $Q$ within the collection of all views.

PROOF. Fixing BCQ $Q$, we show that the d-view corresponding to global $Q$-equivalence is useful for $Q$. Consider two d-instances $\mathcal{D}$ and $\mathcal{D}^{\prime}$ that are globally $Q$-equivalent, and assume $\mathcal{D}$ satisfies $Q$. We will transform $\mathcal{D}$ into $\mathcal{D}^{\prime}$ by "swapping one source at a time", replacing the s component of $\mathcal{D}$ with the s component of $\mathcal{D}^{\prime}$. Each swap preserves satisfaction of $Q$ because the s components of $\mathcal{D}$ and $\mathcal{D}^{\prime}$ are (s, $Q$ )-equivalent. Thus $\mathcal{D}^{\prime}$ satisfies $Q$ as well.

To show that the d-view is minimally informative, consider a d-view $\mathcal{V}$ that is useful for $Q$, and suppose we have $\mathcal{D}_{1}$ which agrees with $\mathcal{D}_{2}$ on $\mathcal{V}$. Fixing any source $\mathrm{s}$ we will show that $\mathcal{D}_{1}^{\mathrm{s}}$ and $\mathcal{D}_{1}^{\mathrm{s}}$ are $(\mathrm{s}, Q)$ equivalent. This will show that $\mathcal{D}_{1}$ and $\mathcal{D}_{2}$ are globally $Q$-equivalent, which will imply minimal informativeness. To see this last statement fix any context $C$, and note that $\left(\mathcal{D}_{1}^{\mathrm{s}}, C\right)$ and $\left(\mathcal{D}_{2}^{\mathrm{s}}, C\right)$ agree on $\mathcal{V}$, and hence agree on $Q$. This completes the proof.

Note that the result can be seen as an analog of Theorem 3.1. From the result above we conclude an analog of Corollary 3.5:

PROPOSITION 4.2. If there is any d-view that is useful for $B C Q Q$ and non-disclosing for $B C Q p$, then the $d$-view given by global $Q$-equivalence is useful for $Q$ and nondisclosing for $p$.

From an ECR to a concrete d-view. We now have a useful d-view that is minimally informative within the set of all d-views, but it is given only as the ECR global $Q$ equivalence, and it is not clear that there are any views in the usual sense - isomorphisminvariant functions producing relations of some fixed schema - that correspond to this 
ECR. Our next goal is to show that global $Q$-equivalence is induced by a d-view defined using standard database queries.

A shuffle of a CQ is a mapping from its free variables to themselves (not necessarily injective). Given a CQ $Q$ and a shuffle $\mu$, we denote by $\mu(Q)$ the CQ that results from replacing each variable occurring in $Q$ by its $\mu$-image. We call $\mu(Q)$ a shuffled query. For example, consider the query $\exists y \cdot R\left(x_{1}, x_{2}, x_{2}, y\right) \wedge S\left(x_{2}, x_{3}, x_{3}, y\right)$. Then, the query $\exists y . R\left(x_{2}, x_{1}, x_{1}, y\right) \wedge S\left(x_{1}, x_{1}, x_{1}, y\right)$ is a shuffle of $Q$.

The canonical context query for $Q$ at source $s, \operatorname{CanCtxt}^{\mathrm{s}}(Q)$, is the CQ whose atoms are all the atoms of $Q$ that are not in source $s$, and whose free variables $\operatorname{are} S J \operatorname{Vars}(\mathrm{s}, Q)$.

Definition 4.3. For a source s, a BCQ $Q$ and a variable binding $\sigma$ for $Q$, a shuffle $\mu$ of $\operatorname{CanView}^{\mathrm{s}}(Q)$ is invariant relative to $\left\langle\sigma, \operatorname{CanCtxt}^{\mathrm{s}}(Q)\right\rangle$ if for any d-instance $\mathcal{D}$ such that $\mathcal{D}, \sigma \mid=\operatorname{CanCtxt}^{\mathrm{s}}(Q)$, we have $\mathcal{D}, \sigma \mid=\mu\left(\operatorname{CanCtxt}^{\mathrm{s}}(Q)\right)$. When the source and the utility query is clear, we refer to $\mu$ simply as an invariant shuffle for brevity.

Note that we can verify this invariance by finding a homomorphism going from $\sigma\left(\mu\left(\operatorname{CanCtxt}^{\mathrm{s}}(Q)\right)\right)$ to $\sigma\left(\mathrm{CanCtxt}^{\mathrm{s}}(Q)\right)$.

Invariance concerns every binding $\sigma$. We would like to abstract to bindings satisfying a set of equalities. A type for $S J \operatorname{Vars}(\mathrm{s}, Q)$ is a set of equalities between variables in $\operatorname{SJVars}(\mathrm{s}, Q)$. The notion of a variable binding satisfying a type is the standard one. For a type $\tau$, we can talk about a mapping $\mu$ being invariant relative to $\left\langle\tau, \operatorname{CanCtxt}^{s}(Q)\right\rangle$ : the invariance condition holds for all bindings $\sigma$ satisfying $\tau$.

Example 4.4. We illustrate the notion of invariant shuffle with an example that will become important later.

Consider a d-schema with two sources, one containing a ternary relation name $R$ and the other containing a unary relation name $S$. Consider the utility query $Q$ :

$$
\exists x_{1}, x_{2}, y \cdot R\left(x_{1}, x_{2}, y\right) \wedge R\left(y, x_{2}, x_{1}\right) \wedge S\left(x_{1}\right) \wedge S\left(x_{2}\right)
$$

The source-join variables in each source are $x_{1}$ and $x_{2}$. Let $\tau$ be the type that states $x_{1} \neq x_{2}$. Let us consider the invariant shuffles for the $R$-source and $\tau$. The canonical context query of $Q$ for this source is $S\left(x_{1}\right) \wedge S\left(x_{2}\right)$.

The identity is always an invariant shuffle. We claim that the mapping $\mu_{0}$ taking $x_{1}$ and $x_{2}$ both to $x_{1}$ is an invariant shuffle for $\tau$. Suppose we have a $d$-instance $\mathcal{D}$ and a binding $\sigma$ to the source join variables that is consistent with $\tau$ and such that $\mathcal{D}, \sigma \mid=S\left(x_{1}\right) \wedge S\left(x_{2}\right)$. Applying $\mu_{0}$ to $S\left(x_{1}\right) \wedge S\left(x_{2}\right)$ gives $S\left(x_{1}\right)$, and clearly we have $\mathcal{D}, \sigma=S\left(x_{1}\right)$. Thus the shuffle $\mu_{0}$ is invariant. Similarly we can see that the mapping sending $x_{1}$ and $x_{2}$ to $x_{2}$ is invariant, as well as the mapping that swap $x_{1}$ and $x_{2}$.

Summing up, an invariant shuffle for a source s and type $\tau$ is a symmetry of the query formed from the utility query by specializing to type $\tau$ and restricting to the atoms lying outside of s. In this example, the utility query outside of the $R$-source is extremely symmetric, so it has the maximal set of invariant shuffles.

For a source $s$ and a CQ $Q$, let $\tau_{1}, \ldots, \tau_{n}$ be all the equality types over the variables in $\operatorname{SJVars}(\mathrm{s}, Q)$.

Definition 4.5. The set of invariant shuffle views of $Q$ for source s consist of views $V_{\tau_{1}}, \ldots, V_{\tau_{n}}$ where each $V_{\tau_{i}}$ is defined as $\tau_{i}(\mathbf{x}) \wedge \bigvee_{\mu} \mu\left(\operatorname{CanView}^{\mathrm{s}}(Q)\right)$, where $\mathbf{x}$ are the source-join variables of $Q$ for source $\mathrm{s}$, and where the disjunction is over shuffles invariant relative to $\tau_{i}$. 
Note that since the domain of $\mu$ is finite, there are only finitely many mappings on them, and thus there are finitely many disjuncts in each view up to equivalence.

We can show that global $Q$ equivalence corresponds to agreement on these views:

Proposition 4.6. For any BCQ $Q$ and any source s, two s-instances are $(s, Q)$ equivalent if and only if they agree on each invariant shuffle view of $Q$ for $s$.

The proof will go through an intermediate view, also given by an ECR.

Definition 4.7. Given two s-instances $\mathcal{I}_{1}$ and $\mathcal{I}_{2}$, we say that $\mathcal{I}_{1}$ and $\mathcal{I}_{2}$ are invariant shuffle equivalent (relative to $Q$ ) if: Whenever $\mathcal{I}_{1}, \sigma$ satisfies $\operatorname{CanView}^{\mathrm{s}}(Q)(\mathbf{x})$ then there is some shuffle $\mu$ invariant relative to $\left\langle\sigma, \operatorname{CanCtxt}^{\mathrm{s}}(Q)\right\rangle$ such that $\mathcal{I}_{2}, \sigma$ satisfies $\mu\left(\operatorname{CanView}^{\mathrm{s}}(Q)\right)(\mathbf{x})$, and similarly with the role of $\mathcal{I}_{1}$ and $\mathcal{I}_{2}$ reversed.

We show:

PROPOSITION 4.8. For any source s, invariant shuffle equivalence is identical to $(\mathrm{s}, Q)$-equivalence.

PROOF. First, suppose $\mathcal{I}_{1}, \mathcal{I}_{2}$ are local instances for source s that are invariant shuffle equivalent, and suppose we have a match of $Q$ in $\left(\mathcal{I}_{1}, C\right)$ via $h^{1, C}$. We want to show that there is a match in $\left(\mathcal{I}_{2}, C\right)$.

We know that the variables in SJVars $(\mathrm{s}, Q)$ are mapped by $h^{1, C}$ into $\mathcal{I}_{1}$. Let $h_{0}$ be the restriction of $h^{1, C}$ to the variables of SJVars $(\mathrm{s}, Q)$. Then $\mathcal{I}_{1}, h_{0}$ satisfies $\operatorname{CanView}^{\mathrm{s}}(Q)$. Thus by shuffle equivalence there is some shuffle $\mu$ invariant relative to $\left\langle h_{0}\right.$, $\left.\operatorname{CanView}^{\mathrm{s}}(Q)\right\rangle$, such that $\mathcal{I}_{2}, h_{0}=\mu\left(\operatorname{CanView}^{\mathrm{s}}(Q)\right)$, with witness $h_{2}$ extending $h_{0}$. We also know that $C, h_{0}$ satisfies $\operatorname{CanCtxt}^{\mathrm{S}}(Q)$, since $h^{1, C}$ witnesses this as well. Applying the definition of shuffle invariance, $C, h_{0}$ satisfies $\mu\left(\operatorname{CanCtxt}^{\mathrm{s}}(Q)\right)$. Let $h^{\mu, C}$ be a homomorphism witnessing this. Note that since $h^{\mu, C}$ extends $h_{0}$ and $h_{0}$ restricts $h^{1, C}, h^{\mu, C}$ and $h^{1, C}$ agree on their common variables. Define $h^{2, C}$ by mapping the variables in $\operatorname{SVars}(\mathrm{s}, Q)$ as in $h_{2}$, and those variables outside of SJVars $(\mathrm{s}, Q)$ as in $h^{\mu, C}$. Since these are two compatible homomorphisms, $h^{2, C}$ witnesses that $\left(\mathcal{I}_{2}, C\right) \models Q$. This completes the argument that invariant shuffle equivalence implies global $Q$-equivalence.

We now show that global $Q$-equivalence implies shuffle equivalence. Suppose $s$ instances $\mathcal{I}_{1}, \mathcal{I}_{2}$ are globally $Q$-equivalent, and $\mathcal{I}_{1}, \sigma$ satisfies $\operatorname{CanView}^{\mathrm{s}}(Q)(\mathbf{x})$, We will show that there is a shuffle $\mu$, invariant relative to $\left\langle\sigma, \operatorname{CanCtxt}^{\mathrm{s}}(Q)\right\rangle$, such that $\mathcal{I}_{2}, \sigma \mid=\mu\left(\operatorname{CanView}^{\mathrm{s}}(Q)\right)(\mathbf{x})$. Let $C_{1}$ be the canonical database of $\sigma\left(\operatorname{CanCtxt}^{\mathrm{s}}(Q)\right)$. That is, for each source $\mathrm{s}^{\prime}$ other than $\mathrm{s}$, we have a fact for each $\mathrm{s}^{\prime}$ atom of $Q$, where each variable $x$ of $\operatorname{SJVars}(\mathrm{s}, Q)$ is replaced by $\sigma(x)$ and each variable $x$ not in $\operatorname{SJVars}(\mathrm{s}, Q)$ is replaced by a fresh element $c_{x}$.

$Q$ clearly holds in $\left(\mathcal{I}_{1}, C_{1}\right)$. So by global $Q$-equivalence, $Q$ holds in $\left(\mathcal{I}_{2}, C_{1}\right)$ via some homomorphism $h$. Note that, by the design of $C_{1}$, the only elements that are shared between $\mathcal{I}_{2}$-facts and $C_{1}$-facts lie in the range of $\sigma$. Thus $h$ must map the variables in SJVars (s, $Q)$ to the image of $\sigma$. The binding $\sigma$ may not be injective, but we let $\sigma^{-1}$ be a "right inverse" that is, any function from the range of $\sigma$ to variables such that for any $c$ in the range of $\sigma \sigma\left(\sigma^{-1}(c)\right)=c$. Let $\mu$ map any variable $x \in \operatorname{SJVars}(\mathrm{s}, Q)$ to $\sigma^{-1}(h(x))$. So $\sigma(\mu(x))=h(x)$.

We first claim that $\mu$ is invariant relative to $\left\langle\sigma, \operatorname{CanCtxt}^{\mathrm{s}}(Q)\right\rangle$. We show this by arguing that $h$ is a homomorphism from $\sigma\left(\mu\left(\operatorname{CanCtxt}^{\mathrm{s}}(Q)\right)\right)$ to $\sigma\left(\operatorname{CanCtxt}^{\mathrm{s}}(Q)\right)$. By 
definition of $\mu$, we have for each atom $A\left(x_{1} \ldots x_{m}, y_{1} \ldots y_{n}\right)$ of $\operatorname{CanCtxt}^{\mathrm{s}}(Q)(\mathbf{x})$,

$$
\begin{array}{r}
A\left(h\left(x_{1}\right) \ldots h\left(x_{m}\right), h\left(y_{1}\right) \ldots h\left(y_{n}\right)\right)= \\
A\left(\sigma\left(\mu\left(x_{1}\right)\right) \ldots \sigma\left(\mu\left(x_{m}\right)\right), h\left(y_{1}\right) \ldots h\left(y_{n}\right)\right)
\end{array}
$$

$A\left(h\left(x_{1}\right) \ldots h\left(x_{m}\right), h\left(y_{1}\right) \ldots h\left(y_{n}\right)\right)$ is in $\sigma\left(\operatorname{CanCtxt}^{\mathrm{s}}(Q)\right)$ since by assumption $h$ is a homomorphism from $Q$ to $\left(\mathcal{I}_{2}, C_{1}\right), C_{1}$ is the canonical database of $\sigma\left(\operatorname{CanCtxt}^{\mathrm{s}}(Q)\right)$, and $\mathcal{I}_{2}$ is an $s$-instance, and hence cannot contain any facts over the relations in $\left(\mu\left(\operatorname{CanCtxt}^{\mathrm{s}}(Q)\right)\right)(\sigma)$. Thus

$$
A\left(\sigma\left(\mu\left(x_{1}\right)\right) \ldots \sigma\left(\mu\left(x_{m}\right)\right), h\left(y_{1}\right) \ldots h\left(y_{n}\right)\right)
$$

lies in $\sigma(\operatorname{CanCtxt}(Q))$ as required.

We next claim that $\mathcal{I}_{2}, \sigma=\mu\left(\operatorname{CanView}^{\mathrm{s}}(Q)\right)$. The witness will be the extension $h^{\prime}$ of $\sigma$ that maps all variables in $\operatorname{SVars}(s, Q)-\operatorname{SJVars}(\mathrm{s}, Q)$ via $h$. Consider an atomic formula $A\left(x_{1} \ldots x_{m}, y_{1} \ldots y_{n}\right)$ of $\mathrm{CanView}^{\mathrm{s}}(Q)$, where $\mathbf{x}$ are free variables of $\mathrm{CanView}^{\mathrm{s}}(Q)$. Therefore $A\left(\mu\left(x_{1}\right) \ldots \mu\left(x_{m}\right), y_{1} \ldots y_{n}\right)$ is a generic atom of $\mu\left(\operatorname{CanView}^{\mathrm{s}}(Q)\right)$. To argue that $h^{\prime}$ is a homomorphism that witnesses $\mathcal{I}_{2}, \sigma=\mu\left(\mathrm{CanView}^{\mathrm{s}}(Q)\right)$, we need to argue that

$$
A\left(\sigma\left(\mu\left(x_{1}\right)\right) \ldots \sigma\left(\mu\left(x_{m}\right)\right), h\left(y_{1}\right) \ldots h\left(y_{n}\right)\right)
$$

holds in $\mathcal{I}_{2}$.

But by the definition of $\mu$, this is equivalent to showing that

$$
A\left(h\left(x_{1}\right) \ldots h\left(x_{m}\right), h\left(y_{1}\right) \ldots h\left(y_{n}\right)\right)
$$

holds in $\mathcal{I}_{2}$. But this follows since $h$ is a homomorphism of $Q$ into $\left(\mathcal{I}_{2}, C_{1}\right)$.

To complete the proof of Proposition 4.6, we show:

Proposition 4.9. For any $C Q Q$ and source s, two s-instances are invariant shuffle equivalent if and only if they agree on each invariant shuffle view of $Q$ for $s$.

PROOF. We first show that if $\mathcal{I}_{1}$ and $\mathcal{I}_{2}$ agree on the invariant shuffle views of $Q$, they are invariant shuffle equivalent. Suppose $\mathcal{I}_{1}, \sigma \quad=\operatorname{CanView}^{\mathrm{s}}(Q)$, and let $\tau$ be the type of $\sigma$. Since the identity is invariant relative to $\tau$, we have $\mathcal{I}_{1}, \sigma$ satisfies $V_{\tau}$, and thus $\mathcal{I}_{2}, \sigma$ must satisfy it. Therefore there is $\mu$ that is invariant relative to $\tau$ such that $\mathcal{I}_{2}, \sigma \mid=\mu\left(\mathrm{CanView}^{\mathrm{s}}(Q)\right)$. Since $\tau$ is of type $\sigma$, we have $\mu$ is invariant relative to $\left\langle\sigma, \operatorname{CanCtxt}^{s}(Q)\right\rangle$. Arguing symmetrically for $\mathcal{I}_{2}$, we see that $\mathcal{I}_{1}$ and $\mathcal{I}_{2}$ are invariant shuffle equivalent.

In the other direction, suppose $\mathcal{I}_{1}$ and $\mathcal{I}_{2}$ are invariant shuffle equivalent. We will argue that they agree on each invariant shuffle view $V_{\tau}$.

Towards that end, suppose $\mathcal{I}_{1}, \sigma \mid=V_{\tau}$. That is, $\mathcal{I}_{1}, \sigma \mid=\tau \wedge \mu\left(\operatorname{CanView}^{\mathrm{s}}(Q)\right)$ for some $\mu$ that is invariant relative to $\tau$. Let $\sigma^{\prime}$ be the pre-image of $\sigma$ under $\mu$ : that is, the variable binding defined by $\sigma^{\prime}(x)=\sigma(\mu(x))$. Then $\mathcal{I}_{1}, \sigma^{\prime} \mid=$ CanView $^{\mathrm{s}}(Q)$ by definition. Thus by invariant shuffle equivalence, there is $\mu^{\prime}$ invariant for $\sigma^{\prime}, \operatorname{CanCtxt}^{s}(Q)$ such that $\mathcal{I}_{2}, \sigma^{\prime}=\mu^{\prime}\left(\operatorname{CanView}^{\mathrm{s}}(Q)\right)$.

Let $\mu^{\prime \prime}=\mu^{\prime}(\mu)$. We will show that $\mu^{\prime \prime}$ witnesses that $\mathcal{I}_{2}, \sigma \mid=V_{\tau}$. We first verify invariance:

Claim 1. $\mu^{\prime \prime}$ is invariant relative to $\tau, \operatorname{CanCtxt}{ }^{\mathrm{s}}(Q)$. 
Proof. Suppose $\sigma_{0}$ satisfies $\tau$, and $\mathcal{I}_{0}, \sigma_{0}=\operatorname{CanCtxt}{ }^{\mathrm{s}}(Q)$. Let $\sigma_{0}^{\prime}$ be the pre-image of $\sigma^{\prime}$ under $\mu$. Note that a shuffle that is invariant for $\sigma$ must be invariant for $\sigma_{0}$, since $\sigma_{0}$ satisfies all the equalities that $\sigma$ does. Similarly, a shuffle that is invariant for $\sigma^{\prime}$ must be invariant for $\sigma_{0}^{\prime}$. We can now make the following chain of conclusions, with the rationale appearing to the right of each statement:

$$
\begin{array}{r}
\mathcal{I}_{0}, \sigma_{0}=\mu\left(\operatorname{CanCtxt}^{\mathrm{s}}(Q)\right) \text { by invariance of } \mu \text { for } \tau \\
\mathcal{I}_{0}, \sigma_{0}^{\prime}=\operatorname{CanCtxt}^{\mathrm{s}}(Q) \text { by definition of } \sigma_{0}^{\prime} \\
\mathcal{I}_{0}, \sigma_{0}^{\prime}=\mu^{\prime}\left(\operatorname{CanCtxt}^{\mathrm{s}}(Q)\right) \text { by invariance of } \mu^{\prime} \text { for } \sigma_{0}^{\prime} \\
\mathcal{I}_{0}, \sigma_{0}=\mu^{\prime \prime}\left(\operatorname{CanCtxt}^{\mathrm{s}}(Q)\right) \text { by definition of } \sigma_{0}^{\prime} \text { again }
\end{array}
$$

The last line shows that $\mu^{\prime \prime}$ is invariant as required.

We now show that $\mathcal{I}_{2}, \sigma$ satisfies the corresponding shuffled query.

Claim 2. $\mathcal{I}_{2}, \sigma \mid=\mu^{\prime \prime}\left(\operatorname{CanView}^{\mathrm{s}}(Q)\right)$.

PROOF. For any instance $\mathcal{I}$, bindings $\sigma_{0}$, CQs $R$, and shuffles $\mu_{0}$, let $\sigma_{1}$ be the preimage of $\sigma_{0}$ under $\mu_{0}$. We note that $\mathcal{I}, \sigma_{0}=\mu(R)$ if and only if $\mathcal{I}, \sigma_{1}=R$. This follows just from unwinding the definitions.

Let $\sigma^{\prime \prime}$ be the pre-image of $\sigma$ under $\mu^{\prime \prime}$. Note that $\sigma^{\prime \prime}$ is also the pre-image of $\sigma^{\prime}$ under $\mu^{\prime}$. From the observation just above, we see that the following are equivalent:

$$
\begin{array}{r}
\mathcal{I}_{2}, \sigma \mid=\mu\left(\operatorname{CanView}^{\mathrm{s}}(Q)\right) \\
\mathcal{I}_{2}, \sigma^{\prime \prime} \mid=\operatorname{CanView}^{\mathrm{s}}(Q) \\
\mathcal{I}_{2}, \sigma^{\prime} \mid=\mu^{\prime}\left(\operatorname{CanView}^{\mathrm{s}}(Q)\right)
\end{array}
$$

which gives the proof of the claim.

Combining the two claims, we conclude that $\mathcal{I}_{2}, \sigma$ satisfies $V_{\tau}$ as required, which completes the proof of Proposition 4.9.

Putting together Proposition 4.2 and 4.6, we obtain:

THEOREM 4.10. [Minimally informative d-views for the class of all views] The invariant shuffle views are minimally informative for $Q$ within the class of all views.

This yields a corollary for non-disclosure analogous to Corollary 3.5:

COROLLARY 4.11. If an arbitrary d-view $\mathcal{V}$ is useful for $B C Q Q$ and non-disclosing for $B C Q$ p, then the d-view containing, for each source s, the invariant shuffle views of $Q$ for s, is useful and non-disclosing. In particular, some DCQ is useful for $Q$ and non-disclosing for $p$.

In Example 1.1 there are no nontrivial shuffles, so the canonical d-view is minimally informative within the class of all views. In general, the invariant shuffle views can be unsafe: different disjuncts may contain distinct variables. Of course, they can be implemented easily by using a wildcard to represent elements outside the active domain. Further, we can convert each of these unsafe views to an "information-equivalent" set of relational algebra views: 
Proposition 4.12. For every view defined by a (possibly unsafe) DCQ, there is a finite set of relational algebra-based views $\mathcal{V}^{\prime}$ that induces the same ECR. Applying this to the invariant shuffle views for a $C Q Q$, we can find a relational algebra-based $d$-view that is minimally informative for $Q$ within the class of all views.

The intuition behind the proposition is to construct separate views for different subsets of the variables that occur as a CQ disjunct. A view with a given set of variables $S$ will assert that some CQ disjunct with variables $S$ holds and that no disjunct corresponding to a subset of $S$ holds.

PROOF. Clearly if we have this for a single DCQ view $V$, we obtain it for a finite set of views (and hence for a d-view) by applying the construction to each view in the set.

We consider a DCQ $V\left(x_{1} \ldots x_{n}\right)$ defined by $\bigvee_{i} \varphi_{i}$. For each $\varphi_{i}$ let $\operatorname{Vars}\left(\varphi_{i}\right)$ be the set of variables within it, and for each subset $S$ of the vars let $D_{S}$ be the set of $i$ such that $\varphi_{i}$ uses variables $S$. Given a set of variables $S=x_{j_{1}} \ldots x_{j_{k}}$ with $D_{S} \neq \emptyset$, create a view $V_{S}\left(x_{j_{1}} \ldots x_{j_{k}}\right)$ defined by:

$$
\bigvee_{\left\{\varphi_{i} \mid \operatorname{Vars}\left(\varphi_{i}\right)=S\right\}} \varphi_{i} \wedge \neg\left(\bigvee_{\left\{\varphi_{j} \mid \operatorname{Vars}\left(\varphi_{j}\right) \subsetneq S\right\}} \varphi_{j}\right)
$$

Example 4.13. We explain the construction of relational algebra views by example. Suppose we have a view $V$ given by a DCQ:

$$
R(x, y, z) \vee P(x, y, z) \vee W(x, y, w) \vee T(x, y)
$$

We have three sets $S$ such that $D_{S} \neq \emptyset: S_{1}=\{x, y, z\}, S_{2}=\{x, y, w\}$ and $S_{3}=\{x, y\}$. Our construction will create views for each of these.

View $V_{S_{1}}(x, y, z)$ is defined by query:

$$
[R(x, y, z) \vee P(x, y, z)] \wedge \neg T(x, y)
$$

$V_{S_{2}}(x, y, w)$ is defined by query:

$$
W(x, y, w) \wedge \neg T(x, y)
$$

Finally, $V_{S_{3}}(x, y)$ is defined by the query $T(x, y)$. It is not difficult to see that these views determine $V$ and vice versa.

Returning to the general case, we claim that the set of views $V_{S}$ determines $V$ and vice versa. In one direction, suppose $\mathcal{I}_{1}$ and $\mathcal{I}_{2}$ agree on each $V_{S}$, and $\mathcal{I}_{1} \mid=V(\mathbf{t})$. Choose $i$ with $\mathcal{I}_{1} \mid=\varphi_{i}(\mathbf{t})$ such that $\operatorname{Vars}\left(\varphi_{i}\right)$ is minimal. Let $\mathbf{t}^{\prime}$ be the subtuple of $\mathbf{t}$ corresponding to the variables of $\varphi_{i}$. Then, by minimality of $\operatorname{Vars}\left(\varphi_{i}\right), \mathcal{I}_{1}=\operatorname{Vars}\left(\varphi_{i}\right)\left(\mathbf{t}^{\prime}\right)$ and hence $\mathcal{I}_{2} \mid=V_{\operatorname{Vars}\left(\varphi_{i}\right)}\left(\mathbf{t}^{\prime}\right)$. From this we see that $\mathcal{I}_{2} \mid=\varphi_{j}\left(\mathbf{t}^{\prime}\right)$ with $\operatorname{Vars}\left(\varphi_{j}\right)=\operatorname{Vars}\left(\varphi_{i}\right)$ and hence $\mathcal{I}_{2} \mid=V(\mathbf{t})$.

In the other direction, suppose $\mathcal{I}_{1}$ and $\mathcal{I}_{2}$ agree on $V$, and $\mathcal{I}_{1} \mid=V_{S}(\mathbf{t})$. Fix $\varphi_{i}$ with variables from $S$ such that $\mathcal{I}_{1} \mid=\varphi_{i}(\mathbf{t})$. We need to show $\mathcal{I}_{2} \mid=V_{S}(\mathbf{t})$. We work by induction and assume that $V$ determines $V_{S^{\prime}}$ for each proper subset $S^{\prime}$ of $S$. We know that $\mathcal{I}_{1} \mid=V(\mathbf{t})$. Hence $\mathcal{I}_{2} \mid=V(\mathbf{t})$, and thus there is some $j$ such that $\mathcal{I}_{2} \mid=\varphi_{j}(\mathbf{t})$.

First, consider the case where $S$ consists of all free variables in $V$. In this case the free variables of $\varphi_{j}$ are either the same as $S$, or are a proper subset of $S$, since it cannot be a superset. If $\varphi_{j}$ contains all the variables of $\varphi_{i}$, then $\varphi_{j}$ witnesses the left conjunct 
in $V_{S}$ holding for $\mathbf{t}$. The induction hypothesis implies that the right conjunct is satisfied. We can conclude that $\mathcal{I}_{2} \mid=V_{S}(\mathbf{t})$ as required. If the free variables of $\varphi_{j}$ are a proper subset $S^{\prime}$ of the variables in $S$, then we have $\mathcal{I}_{2} \mid=\varphi_{k}\left(\mathbf{t}^{\prime}\right)$ for $\mathbf{t}^{\prime}$ a proper subtuple of $\mathbf{t}$. Choose $\varphi_{k}$ and $\mathbf{t}^{\prime}$ with this property such that the variables $S^{\prime}$ involved are minimized. The $\mathcal{I}_{2} \mid=V_{S^{\prime}}\left(\mathbf{t}^{\prime}\right)$ so by the induction hypothesis $\mathcal{I}_{1} \mid=V_{S^{\prime}}\left(\mathbf{t}^{\prime}\right)$, which contradicts that $\mathcal{I}_{1} \mid=V_{S}(\mathbf{t})$.

Next, consider the case where $S$ is a proper subset of the variables. We extend $\mathbf{t}$ to $\mathbf{t}^{\prime}$ choosing elements outside the active domain of both $\mathcal{I}_{1}$ and $\mathcal{I}_{2}$. Since $\mathcal{I}_{1}$ with $\mathrm{t}$ satisfies some positive literal in $V_{S}$, we know $\mathcal{I}_{1} \mid=V\left(\mathbf{t}^{\prime}\right)$, and therefore $\mathcal{I}_{2} \mid=V\left(\mathbf{t}^{\prime}\right)$. Thus there is a proper subtuple $\mathbf{t}^{\prime \prime}$ of $\mathbf{t}^{\prime}$ and a disjunct $\varphi_{k}$ such that $\mathcal{I}_{2}=\varphi_{i}\left(\mathbf{t}^{\prime \prime}\right)$. As above, we can choose $t^{\prime \prime}$ minimal. By our choice of the elements in $t^{\prime}-t$, there is $t^{\prime \prime}$ a subtuple of $t$. If $\mathbf{t}^{\prime \prime}=\mathbf{t}$, then we can conclude that $\mathcal{I}_{2} \mid=V_{S}(\mathbf{t})$ as required. If $\mathbf{t}^{\prime \prime}$ is a proper subtuple, we argue by contradiction of the induction hypothesis as above.

Example 4.14. Let us recall the d-schema of Example 4.4, which contained a ternary relation name $R$ and the other containing a unary relation name $S$. Recall that the utility query $Q$ was:

$$
\exists x_{1}, x_{2}, y \cdot R\left(x_{1}, x_{2}, y\right) \wedge R\left(y, x_{2}, x_{1}\right) \wedge S\left(x_{1}\right) \wedge S\left(x_{2}\right)
$$

The canonical view for the $R$ source $\operatorname{CanView}^{R}(Q)$ is $\exists y . R\left(x_{1}, x_{2}, y\right) \wedge R\left(y, x_{2}, x_{1}\right)$.

Observe that $Q$ is a minimal CQ, and hence by Theorem 3.1 the canonical d-view of $Q$ is minimally informative among the CQ-based d-views. We will argue that this d-view is not minimally informative useful for $Q$ among all d-views, by arguing that it discloses more secrets than the shuffle views disclose. Consider the secret query $p=\exists x \cdot R(x, x, x)$. We show that the canonical d-view of $Q$ is UN disclosing for $p$. Consider the critical instance of the $R$-source, which we recall from Definition 2.9. An external party will know that the instance contains $\left\{R\left(*, *, y_{0}\right), R\left(y_{0}, *, *\right)\right\}$ for some $y_{0}$. On the other hand, if $y_{0} \neq *$, then the canonical d-view would reveal $x_{1}=y_{0}, x_{2}=*$. So $y_{0}$ must be $*$, and therefore $p$ is disclosed. By Corollary 3.5, no CQ-based d-view can be UN non-disclosing for $p$ and useful for $Q$.

The shuffle views of $Q$ are always useful for $Q$. We will show that they are UN non-disclosing for $p$. Let us start by deriving the invariant shuffle views for the $R$ source. There are two types, $\tau_{1}$ in which $x_{1}=x_{2}$, and $\tau_{2}$ in which the variables are not identified.

For a binding satisfying $\tau_{1}$, the canonical view of $Q$ for the $R$ source is equivalent to

$$
\exists y \cdot R\left(x_{1}, x_{1}, y\right) \wedge R\left(y, x_{1}, x_{1}\right)
$$

Since there is only one free variable in it, there is only one invariant shuffle, the identity. Thus the corresponding shuffle view for $\tau_{1}$ is:

$$
\mathrm{\vee}_{\tau_{1}}=\exists y \cdot R\left(x_{1}, x_{1}, y\right) \wedge R\left(y, x_{1}, x_{1}\right)
$$

For bindings satisfying $\tau_{2}$ we saw in Example 4.4 that there are several shuffles invariant for $\operatorname{CanCtxt}^{R}(Q)=S\left(x_{1}\right) \wedge S\left(x_{2}\right)$ : the identity, the shuffle which swaps $x_{1}$ and $x_{2}$, the shuffle in which $x_{1}$ and $x_{2}$ both go to $x_{1}$, and the shuffle in which both $x_{1}$ and $x_{2}$ go to 
$x_{2}$. Thus we get the view $\mathrm{V}_{\tau_{2}}$ defined as $x_{1} \neq x_{2}$ conjoined with:

$$
\begin{aligned}
& \exists y \cdot R\left(x_{1}, x_{2}, y\right) \wedge R\left(y, x_{2}, x_{1}\right) \vee \\
& \exists y \cdot R\left(x_{1}, x_{1}, y\right) \wedge R\left(y, x_{1}, x_{1}\right) \vee \\
& \exists y \cdot R\left(x_{2}, x_{2}, y\right) \wedge R\left(y, x_{2}, x_{2}\right) \vee \\
& \exists y \cdot R\left(x_{2}, x_{1}, y\right) \wedge R\left(y, x_{1}, x_{2}\right)
\end{aligned}
$$

This last view is unsafe, but via Proposition 4.12 we can convert it into a safe relational algebra view that yields the same ECR, $\mathrm{V}_{\tau_{2}}^{\text {safe }}$ defined as $x_{1} \neq x_{2}$ conjoined with:

$$
\begin{aligned}
& \neg\left(\exists y \cdot R\left(x_{1}, x_{1}, y\right) \wedge R\left(y, x_{1}, x_{1}\right)\right) \wedge \\
& \neg\left(\exists y \cdot R\left(x_{2}, x_{2}, y\right) \wedge R\left(y, x_{2}, x_{2}\right)\right) \wedge \\
& {\left[\left(\exists y \cdot R\left(x_{1}, x_{2}, y\right) \wedge R\left(y, x_{2}, x_{1}\right) \vee\right.\right.} \\
& \left.\left.\exists y \cdot R\left(x_{2}, x_{1}, y\right) \wedge R\left(y, x_{1}, x_{2}\right)\right)\right]
\end{aligned}
$$

We now argue that the shuffle views are UN non-disclosing for $p$. This is because in any d-instance we can replace each fact $R\left(x_{0}, x_{0}, x_{0}\right)$ by facts $R\left(x_{0}, x_{0}, c\right)$ and $R\left(c, x_{0}, x_{0}\right)$ for a fresh $c$, obtaining an indistinguishable instance where $p$ does not hold. Hence by Proposition 2.5, the canonical views of $Q$ cannot be minimally informative within the class of all views, or even within the class of relational algebra views.

\subsection{The importance of using negation or unsafe queries}

We now consider in a bit more detail which query language features are needed to obtain minimally informative useful d-views.

Let us return to the utility query from Example 4.14:

$$
Q=\exists x_{1}, x_{2}, y_{0} \cdot R\left(x_{1}, x_{2}, y_{0}\right) \wedge R\left(y_{0}, x_{2}, x_{1}\right) \wedge S\left(x_{1}\right) \wedge S\left(x_{2}\right)
$$

with $R$ and $S$ in distinct sources.

Consider the secret query from the same example $p=\exists x \cdot R(x, x, x)$.

We saw from Example 4.14 that there is a d-view that is useful for $Q$ and UN nondisclosing for $p$. Indeed, we have one example of such a d-view which is an unsafe DCQ, and another makes use of negation. We show that this is essential: in this example, we must give up either safety or positivity in order to get a useful and UN non-disclosing $d$-view. Since we know that minimally informative d-views are always minimal with respect to disclosure, this example will also prove that we may need to use either unsafe queries or negation to construct minimally informative $d$-views.

We formalize what we mean by safe and positive views. We recall that a query $Q$ is homomorphism-invariant if $\mu(Q(\mathcal{I})) \subseteq Q\left(\mathcal{I}^{\prime}\right)$ for all pairs of instances $\mathcal{I}$ and $\mathcal{I}^{\prime}$ and all homomorphisms $\mu$ from $\mathcal{I}$ to $I^{\prime}$. A query $Q$ is adom-based when adom $(Q(\mathcal{I})) \subseteq$ $\operatorname{adom}(\mathcal{I})$. Note that all queries defined by CQs and UCQs are homomorphism-invariant and adom-based. As with monotonicity, there are expressive extensions of UCQs that are also homomorphism-invariant. Datalog [1] is again a well-known example of such a language, although we will not need to discuss the details of specific languages that are homomorphism-invariant. A set of views is homomorphism-invariant or adom-based exactly when all its defining queries are. 
We are now ready to state the result formalizing that we cannot avoid either unsafe queries or negation:

Proposition 4.15. Any set of useful views for $Q$ which is homomorphism-invariant and adom-based must UN-disclose p.

Thus in particular, we can protect more information using relational algebra views than by views using only monotone relational algebra operators, even when allowing recursion.

Proof. Let $\mathcal{V}$ be useful for $Q$, homomorphism-invariant and adom-based and $\mathcal{V}_{R}$ be the views on the $R$ sources. Let $\mathcal{D}_{Q}$ be the canonical database of $Q$.

We first claim that the active domain of the view image of $\mathcal{D}_{Q}$ under $\mathcal{V}_{R}$ must contain each of the elements $y_{0}, x_{1}, x_{2}$ :

- If we suppose that $x_{1}$ does not belong to $\operatorname{adom}\left(\mathcal{V}_{R}\left(\mathcal{D}_{Q}\right)\right)$ we can apply a function $h$ mapping $x_{1}$ to another value on the source $R$. By homomorphism-invariance, $\mathcal{V}_{R}$ is not be impacted and, since $\mathcal{D}_{Q}$ and $h\left(\mathcal{D}_{Q}\right)$ agree on the source $S$, we have $\mathcal{V}\left(\mathcal{D}_{Q}\right)=\mathcal{V}\left(h\left(\mathcal{D}_{Q}\right)\right)$. But this contradicts utility of $\mathcal{V}$ for $Q$ as $Q$ holds in $\mathcal{D}_{Q}$ and not in $h\left(\mathcal{D}_{Q}\right)$. Therefore $x_{1}$ needs to appear in adom $\left(\mathcal{V}_{R}\left(\mathcal{D}_{Q}\right)\right)$.

- With the same argument as for $x_{1}, x_{2}$ must also appear in adom $\left(\mathcal{V}_{R}\left(\mathcal{D}_{Q}\right)\right)$.

- Consider the mapping $m$ on the domain of $\mathcal{D}_{Q}$ that swaps $x_{1}$ and $y_{0}$. Thus it produces the canonical database of $\mathcal{D}_{Q^{\prime}}$

$$
\exists x_{1}, x_{2}, y_{0} \cdot R\left(y_{0}, x_{2}, x_{1}\right) \wedge R\left(x_{1}, x_{2}, y_{0}\right) \wedge S\left(y_{0}\right) \wedge S\left(x_{2}\right)
$$

This is an isomorphism, hence a homomorphism mapping $\mathcal{D}_{Q}$ to $\mathcal{D}_{Q^{\prime}}$. The views $\mathcal{V}$ are homomorphism-invariant. Thus, because $x_{1}$ appears in the active domain of $\mathcal{V}\left(\mathcal{D}_{Q^{\prime}}\right), y_{0}$ should also appear in the active domain of $\mathcal{V}\left(\mathcal{D}_{Q^{\prime}}\right.$.

We now consider the critical instance. For this schema, the critical instance is $\mathcal{I}_{0}=$ $\{R(*, *, *), S(*)\}$. Consider an instance $\mathcal{I}^{\prime}$ that is equivalent to $\mathcal{I}_{0}$ on $\mathcal{V}$. Let $\mathcal{I}_{R}^{\prime}$ be the restriction of $\mathcal{I}^{\prime}$ to the $R$-source. Since $\mathcal{V}$ is adom-based, adom $\left(\mathcal{V}_{R}\left(\mathcal{I}_{0}\right)\right)=\{*\}$ and the same holds on $I^{\prime}$.

$\mathcal{I}^{\prime}$ must satisfy $Q$ by usefulness of $\mathcal{V}$ and thus there is a homomorphism $h$ of $\mathcal{D}_{Q}$ into $\mathcal{I}^{\prime}$. Let $x_{1}^{\prime}, x_{2}^{\prime}$, and $y_{0}^{\prime}$ be the images of $x_{1}, x_{2}$ and $y_{0}$ respectively under $h$. Then by homomorphism invariance, $\left\{x_{1}^{\prime}, x_{2}^{\prime}, y_{0}^{\prime}\right\}$ appear in $\operatorname{adom}\left(\mathcal{V}_{R}\left(\mathcal{I}^{\prime}\right)\right)$ but $\operatorname{adom}\left(\mathcal{V}_{R}\left(\mathcal{I}^{\prime}\right)\right)=$ $\{*\}$. Thus all of these must be the same as $*$ and thus $p$ is true in $\mathcal{I}^{\prime}$. This shows that $\mathcal{V}$ is $\mathrm{UN}$-disclosing for $\mathcal{V}$.

\section{LOCAL BACKGROUND KNOWLEDGE}

We now look at the impact of a background knowledge on the sources. We start with the case of a background theory $\Sigma$ in which each sentence is local, referencing relation names within a single source.

\subsection{Extension of results on arbitrary views to the presence of local constraints}

We begin our analysis of the impact of constraints by extending our results on arbitrary d-views to account for local TGDs $\Sigma$. Here we will follow a straightforward extension of the ideas in Section 4.

The notion of a shuffle being $\Sigma$-invariant is defined in the obvious way, restricting to instances that satisfy the constraints in $\Sigma$. The $\Sigma$-invariant shuffle views are also defined 
analogously; they are DCQ views, but can be replaced by the appropriate relational algebra views. By adapting the approach in Section 4, we can show:

THEOREM 5.1. [Minimally informative d-views w.r.t. local TGDs] For any set of local TGDs $\Sigma$, the $\sum$-invariant shuffle views of $Q$ provide a minimally informative useful $d$-view for $Q$ within the class of all views, relative to $\Sigma$.

The result has effective consequences for "tame" TGDs (e.g. with terminating chase) with no non-trivial invariant shuffles. In such cases, the $\Sigma$-invariant shuffle views degenerate to the canonical d-view. From $[7,8]$ we know that checking whether a $\mathrm{d}$-view is UN non-disclosing can is decidable for such classes. Thus we can check whether the canonical d-view is UN non-disclosing effectively, and hence whether any d-view can be useful and UN non-disclosing.

We will now begin the proof of Theorem 5.1. We start by generalizing the ECR global $Q$-equivalence to global $Q$ - $\sum$-equivalence, looking only at contexts that satisfy $\Sigma$. Using the same swapping argument as in Proposition 4.1, we see that the d-view corresponding to this ECR is a minimally informative useful d-view for $Q$ within the class of all views, relative to $\Sigma$.

Let $\sigma$ be a mapping of $\operatorname{SJVars}(s)$ into some instance $\mathcal{I}$. A shuffle $\mu$ of $\operatorname{CanView}^{s}(Q)$ is $\Sigma$-invariant relative to $\left\langle\sigma, \operatorname{CanCtxt}^{s}(Q)\right\rangle$ if whenever $\mathcal{I}^{\prime}, \sigma \mid=\operatorname{CanCtxt}^{s}(Q)$ and $\mathcal{I}^{\prime}$ satisfies $\sum$ then $\mathcal{I}^{\prime}, \sigma=\mu\left(\operatorname{CanCtxt}^{s}(Q)\right)$. Note that since the TGDs are local, the notion of a context satisfying them is well-defined. Invariance is decidable whenever query containment for CQs under $\Sigma$ is decidable; for example, this is the case when $\Sigma$ is a set of dependencies with terminating chase.

Fixing $\Sigma$ and two $s$-instances $\mathcal{I}_{1}$ and $\mathcal{I}_{2}$, we say that $\mathcal{I}_{1}$ and $\mathcal{I}_{2}$ are $\Sigma$-invariant shuffle equivalent if:

- whenever $\mathcal{I}_{1}, \sigma$ satisfies $\operatorname{CanView}^{s}(Q)(\mathbf{x})$ there is some shuffle $\mu$ which is $\Sigma$ invariant relative to $\left\langle\sigma, \operatorname{CanView}^{s}(Q)\right\rangle$, such that $\mathcal{I}_{2}, \sigma \mid=\mu\left(\operatorname{CanView}^{s}(Q)\right)(\mathbf{x})$;

- conversely, whenever $\mathcal{I}_{2}, \sigma$ satisfies $\operatorname{CanView}^{s}(Q)(\mathbf{x})$ there is some shuffle $\mu$ which is $\Sigma$-invariant relative to $\left\langle\sigma, \operatorname{CanView}^{s}(Q)\right\rangle$, such that $\mathcal{I}_{1}, \sigma \mid=\mu\left(\operatorname{CanView}^{s}(Q)\right)(\mathbf{x})$

We can now extend Proposition 4.8, following the same proof:

PROPOSITION 5.2. Suppose $\Sigma$ consists of local TGDs. Then for all instances satisfying the TGDs, $\Sigma$-invariant shuffle equivalence is identical to global $Q-\sum$ equivalence.

PROOF. First, suppose $\mathcal{I}_{1}, \mathcal{I}_{2}$ satisfy all local TGDs and are $\Sigma$-invariant shuffle equivalent. Consider a context $C$ that satisfies local TGDs $\Sigma$ and suppose we have a match of $Q$ in $\left(\mathcal{I}_{1}, C\right)$ via $h^{1, C}$. We want to show that there is a match in $\left(\mathcal{I}_{2}, C\right)$. This will be exactly as in the case without constraints.

We know that the variables in $\operatorname{SJVars}(s, Q)$ are mapped by $h^{1, C}$ into $\mathcal{I}_{1}$. Let $h_{0}$ be the restriction of $h^{1, C}$ to the variables of $\operatorname{SJVars}(s, Q)$. Then $\mathcal{I}_{1}, h_{0}$ satisfies $\operatorname{CanView}^{s}(Q)$. Thus by $\sum$-invariant shuffle equivalence there is a shuffle $\mu$ which is $\sum$-invariant relative to $\left\langle h_{0}\right.$, $\left.\operatorname{CanView}^{s}(Q)\right\rangle$, such that $\mathcal{I}_{2}, h_{0}=\mu\left(\operatorname{CanView}^{s}(Q)\right)$, with witness $h_{2}$ extending $h_{0}$. We also know that $C, h_{0}$ satisfies $\operatorname{CanCtxt}^{s}(Q)$, since $h^{1, C}$ witnesses this as well. Applying the definition of $\Sigma$-invariance, and noting that $C$ satisfies $\Sigma$ by assumption, we infer that $C, h_{0}$ satisfies $\mu\left(\operatorname{CanCtxt}^{s}(Q)\right)$. Let $h^{\mu, C}$ be a homomorphism witnessing this. Note that since $h^{\mu, C}$ extends $h_{0}$ and $h_{0}$ restricts $h^{1, C}, h^{\mu, C}$ and $h_{0}$ agree on their 
common variables. Define $h^{2, C}$ by mapping the variables in $\operatorname{SVars}(s, Q)$ as in $h_{2}$, and those variables outside of $\operatorname{SJVars}(s, Q)$ as in $h^{\mu, C}$. Since these are two compatible homomorphisms, $h^{2, C}$ witnesses that $\left(\mathcal{I}_{2}, C\right) \models Q$. This completes the argument that $\Sigma$-invariant shuffle equivalence implies $Q$ - $\Sigma$-equivalence.

We now show that global $Q$ - $\sum$-equivalence implies $\Sigma$-invariant shuffle equivalence. Suppose $s$-instances $\mathcal{I}_{1}, \mathcal{I}_{2}$ are $Q$ - $\sum$-equivalent, and $\mathcal{I}_{1}, \sigma$ satisfies $\operatorname{CanView}^{s}(Q)(\mathbf{x})$, We will show that there is a shuffle $\mu, \Sigma$-invariant relative to $\left\langle\sigma, \operatorname{CanCtxt}^{s}(Q)\right\rangle$ such that $\mathcal{I}_{2}, \sigma \mid=\mu\left(\operatorname{CanView}^{s}(Q)\right)(\mathbf{x})$.

Let $C_{1}$ be the context defined in two steps. We first proceed as in the case without constraints: for each source $s$ other than $s$, we have a fact for each $s$ atom of $Q$, where each variable $x$ of $\operatorname{SJVars}(s, Q)$ is replaced by $\sigma(x)$ and each variable $x$ not in SJVars is replaced by a fresh element $c_{x}$. In the second step, we perform the chase construction with $\Sigma$ to get an instance that satisfies the local constraints.

$Q$ clearly holds in $\left(\mathcal{I}_{1}, C_{1}\right)$. So by $Q$ - $\sum$-equivalence, $Q$ holds in $\left(\mathcal{I}_{2}, C_{1}\right)$ via some homomorphism $h$. As before, the only elements shared between $\mathcal{I}_{2}$ and $C_{1}$ lie in the range of $\sigma$. Thus $h$ must map the variables in $\operatorname{SJVars}(s, Q)$ to the image of $\sigma$. For each $c$ in the image of $\sigma$, choose a variable $v_{c}$ such that $\sigma$ maps $v_{c}$ to $c$. Let $\mu$ map any variable $x \in \operatorname{SJVars}(s, Q)$ to $v_{h(x)}$. Thus $\sigma(\mu(x))=h(x)$.

We claim that $\mu$ is $\Sigma$-invariant relative to $\left\langle\sigma, \operatorname{CanCtxt}^{s}(Q)\right\rangle$. We show this by arguing that $h$ is a homomorphism from $\mu\left(\operatorname{CanCtxt}^{s}(Q)\right)(\sigma)$ to the chase under $\Sigma$ of CanCtxt ${ }^{s}(Q)(\sigma)$. By definition of $\mu$, we have for each atom $A\left(x_{1} \ldots x_{m}, y_{1} \ldots y_{n}\right)$ of $\operatorname{CanCtxt}^{s}(Q)(\mathbf{x})$,

$$
\begin{gathered}
A\left(h\left(x_{1}\right) \ldots h\left(x_{m}\right), h\left(y_{1}\right) \ldots h\left(y_{n}\right)\right)= \\
A\left(\sigma\left(\mu\left(x_{1}\right)\right) \ldots \sigma\left(\mu\left(x_{m}\right)\right), h\left(y_{1}\right) \ldots h\left(y_{n}\right)\right)
\end{gathered}
$$

$A\left(h\left(x_{1}\right) \ldots h\left(x_{m}\right), h\left(y_{1}\right) \ldots h\left(y_{n}\right)\right)$ is in $\operatorname{Chase}_{\Sigma}\left(\operatorname{CanCtxt}^{s}(Q)(\sigma)\right)$ since $h$ is a homomorphism into $\left(\mathcal{I}_{2}, C_{1}\right)$ and thus for facts in $\mu\left(\operatorname{CanCtxt}^{s}(Q)\right)(\sigma)$, it must map into $C_{1}$.

Thus we conclude

$$
\begin{array}{r}
A\left(\sigma\left(\mu\left(x_{1}\right)\right) \ldots \sigma\left(\mu\left(x_{m}\right)\right), h\left(y_{1}\right) \ldots h\left(y_{n}\right)\right) \\
\in \operatorname{Chase}_{\Sigma}\left(\operatorname{CanCtxt}^{s}(Q)(\sigma)\right)
\end{array}
$$

This completes the proof that $h$ is a homomorphism into the chase, and thus the proof that $\mu$ is $\sum$-invariant relative to $\left\langle\sigma, \operatorname{CanCtxt}^{s}(Q)\right\rangle$.

We next claim that $\mathcal{I}_{2}, \sigma=\mu\left(\operatorname{CanView}^{s}(Q)\right)$. The witness will again be the extension of $\sigma$ that maps all variables in $\operatorname{SVars}(s, Q)-\operatorname{SJVars}(s, Q)$ via $h$.

Consider an atomic formula $A\left(x_{1} \ldots x_{m}, y_{1} \ldots y_{n}\right)$ of $\operatorname{CanView}^{s}(Q)$ where $\mathbf{x}$ are free variables of $\mathrm{CanView}^{s}(Q)$. That is,

$$
A\left(\mu\left(x_{1}\right) \ldots \mu\left(x_{m}\right), \mathbf{y}\right)
$$

is a generic atom of $\mu\left(\mathrm{CanView}^{s}(Q)\right)$. We know that $\left.A\left(h\left(x_{1}\right) \ldots h\left(x_{m}\right), h\left(y_{1}\right) \ldots h\left(y_{n}\right)\right)\right)$ holds in $\mathcal{I}_{2}$, since $h$ is a homomorphism into $\mathcal{I}_{2}$. Thus

$$
\left.A\left(v_{h\left(x_{1}\right)}, \ldots, v_{h\left(x_{m}\right)}, h\left(y_{1}\right) \ldots h\left(y_{n}\right)\right)\right)
$$

holds of $\sigma$ in $\mathcal{I}_{2}$, by definition of $v_{c}$. From this we see that

$$
\left.A\left(\mu\left(x_{1}\right) \ldots \mu\left(x_{m}\right), h\left(y_{1}\right) \ldots h\left(y_{n}\right)\right)\right)
$$

holds of $\sigma$ in $\mathcal{I}_{2}$ as required. 
Recall that the $\sum$-invariant shuffle views of $Q$ for $s$ and types $\tau$ are defined analogously to the case without local TGDs, as $\tau(\mathbf{x}) \wedge \bigvee_{\mu} \mu\left(\operatorname{CanView}^{s}(Q)\right)$ where the disjunction is over $\Sigma$-invariant shuffles of $\tau$.

The following result is proven verbatim as in the case without background knowledge, just adding in the statement that the instances in question satisfy $\Sigma$.

Proposition 5.3. For any Boolean $C Q Q$, and any source s, two s-instances are $\sum$-invariant shuffle equivalent if and only if they agree on each $\Sigma$-invariant shuffle view of $Q$ for $s$.

Putting the prior results together gives us the proof of Theorem 5.1.

\subsection{Extension of results on $C Q$ views to local background knowledge}

We now turn to the impact of local constraints on view design with CQ views. It is easy to show that we cannot generalize the prior results for CQ views to arbitrary local background knowledge. Intuitively using such knowledge we can encode design problems for arbitrary views using CQ views.

Example 5.4. Consider the schema, utility query $Q$ and secret query $p$ from Example 4.14. Let $\Sigma$ consist of the view definitions for the views $\mathrm{V}_{\tau_{1}}$ and $\mathrm{V}_{\tau_{2}}^{\text {safe }}$ in the example. Relative to $\Sigma$, we have CQ view definitions that are useful and UN non-disclosing: these would be views that export the relations $\mathrm{V}_{\tau_{1}}$ and $\mathrm{V}_{\tau_{2}}^{\text {safe }}$, along with the view that exports the interpretation of the additional relation name $S$. However, as observed in Example 4.14, the canonical views of $Q$ are $\mathrm{UN}$ disclosing for $p$.

Thus we restrict to local constraints $\Sigma$ that are TGDs. We show that the results on CQ views extend to this setting. We must now consider utility queries $Q$ that are minimal with respect to $\Sigma$, meaning that there is no strict subquery equivalent to $Q$ under $\Sigma$. We will show the following extension of Theorem 3.1:

THEOREM 5.5. [Minimally informative CQ views w.r.t. local TGDs] Let $\Sigma$ be a set of local TGDs, $Q$ a CQ minimal with respect to $\Sigma$. Then the canonical d-view of $Q$ is minimally informative useful within the class of $C Q$ views relative to $\Sigma$.

If the constraints have terminating chase, then the same holds when the notion of usefulness is relativized to finite instances.

It is possible to prove Theorem 5.5 through the same technique as Theorem 3.1. But we will give an alternative proof. Notice that if there are no constraints, the requirement of terminating chase is vacuous. Thus this represents an alternate proof of Theorem 3.1, which also covers the case where usefulness is relativized to finite instances.

The alternative approach makes use of the following result about TGDs:

PROPOSITION 5.6. If we have a set of TGDs $\Sigma$, a conjunction of atoms $\varphi(\mathbf{x})$ and a disjunction of CQs $\bigvee_{i} Q_{i}$ such that $\Sigma$ entails $\forall \mathbf{x} \varphi(\mathbf{x}) \rightarrow \bigvee_{i} Q_{i}$. Then, for some $i$, $\Sigma$ entails $\forall \mathbf{x} \varphi(\mathbf{x}) \rightarrow Q_{i}$. The same holds when entailment is considered over finite instances only.

This is proven using a simple product construction. A proof can be found in e.g. Lemma 3.5 of [5]. 
We now prove Theorem 5.5. We first prove it for our default notion of usefulness, involving quantification over all instances. Suppose $\mathcal{V}$ is a CQ-based d-view that is useful for $Q$. We show that each view $\mathcal{V}_{s}$ in $\mathcal{V}$ is determined by the canonical d-view.

Fix a source s. Let $\Sigma_{\mathcal{V}, \mathrm{s}}$ be the conjunction of $\Sigma, \Sigma^{\prime}$ a copy of $\Sigma$ on primed relation names, and:

$$
\forall \mathbf{x} . \mathcal{V}_{\mathrm{s}}(\mathbf{x}) \leftrightarrow \mathcal{V}_{\mathrm{s}}^{\prime}(\mathbf{x})
$$

where $\mathcal{V}_{\mathrm{s}}^{\prime}$ is a primed copy of the definitions in $\mathcal{V}_{\mathrm{s}}$.

We know that $\mathcal{V}_{\mathrm{s}}$ determines $\Sigma$-invariant shuffle equivalence on s. Writing this out as an entailment, we obtain:

$$
\Sigma_{\mathcal{V}, \mathrm{s}}=\forall \mathbf{z} . \operatorname{CanView}_{\mathrm{s}}(Q)(\mathbf{z}) \rightarrow \bigvee_{\mu} \operatorname{CanView}_{\mathrm{s}}\left(Q^{\prime}\right)(\mu(\mathbf{z}))
$$

In the disjunction above, $\mu$ varies over shuffles of $\mathrm{s}$ that are $\Sigma$-invariant.

Note that this is an entailment that matches the requirements of Proposition 5.6. So we can apply Proposition 5.6 to infer that there is some $\Sigma$-invariant shuffle $\mu$ such that

$$
\Sigma_{\mathcal{V}, \mathrm{s}} \mid=\forall \mathrm{z} . \operatorname{CanView}_{\mathrm{s}}(Q)(\mathbf{z}) \rightarrow \operatorname{CanView}_{\mathrm{s}}\left(Q^{\prime}\right)(\mu(\mathbf{z}))
$$

We consider two cases, as in the proof of Theorem 3.1:

Case 1: $\mu$ is not injective. Note that the entailment holds for all instances satisfying $\Sigma_{\mathcal{V} \text {, s }}$, and in particular to the case where the primed and unprimed copies of the relations are identical. Thus we have

$$
\Sigma \mid=\forall \mathbf{z} . \operatorname{CanView}_{\mathbf{s}}(Q)(\mathbf{z}) \rightarrow \operatorname{CanView}_{\mathbf{s}}(Q)(\mu(\mathbf{z}))
$$

Consider the mapping on all variables of $Q$ that applies $\mu$ on $\operatorname{SJVars}(Q, \mathrm{~s})$ but is the identity otherwise. We can check that this is a homomorphism of $Q$ into a proper subset of itself. This contradicts the minimality of $Q$ with respect to $\Sigma$, since the analog of Lemma 3.2 holds in the presence of constraints.

Case 2: $\mu$ is bijective. Then some iterate of it is the identity, and thus we can assume that $\mu$ is the identity. That is:

$$
\Sigma_{\mathcal{V}, s} \wedge \operatorname{CanView}_{s}(Q)(\mathbf{z}) \vDash \operatorname{CanView}_{s}\left(Q^{\prime}\right)(\mathbf{z})
$$

But then $\mathcal{V}$ determines the canonical view of $\mathrm{s}$ for $Q$.

The proof of the statement quantifying over finite instances is identical. Note that since we assume that the constraints have terminating chase, $\Sigma$-invariance over finite instances and $\Sigma$-invariance over arbitrary instances is the same, since it is a statement that is captured by a conjunctive query containment using $\Sigma$. If there is a counterexample to such a statement, it can always be taken to be the result of chasing a canonical database. The remaining components of the argument (e.g. Proposition 5.6) hold for quantification over finite instances.

This completes the argument for Theorem 5.5.

The analog of Corollary 3.5 follows from the theorem:

COROLlaRY 5.7. If any $C Q$ based d-view is useful for $\Sigma$-minimal $Q$ and nondisclosing for $p$ relative to $\Sigma$, then the canonical $d$-view of $Q$ is useful for $Q$ and non-disclosing for p relative to $\Sigma$. 
Consequences for decidability. Theorem 5.5 shows that even in the presence of arbitrary local TGDs $\Sigma$ it suffices to minimize the utility query under $\Sigma$ and check the canonical d-view for non-disclosure under $\Sigma$. For arbitrary TGDs, even CQ minimization is undecidable. But for well-behaved classes of TGDs (e.g. those with terminating chase $[3,14]$, or frontier-guarded TGDs [4]) we can perform both minimization and UN non-disclosure checking effectively [7].

Example 5.8. Now that we have our results on local constraints, let us revisit Example 1.5 , in which we had the local constraint $\Sigma$ :

$$
\forall x, y \cdot \operatorname{geolink}(x, y) \leftrightarrow \operatorname{geolink}(y, x)
$$

and the utility query $Q$ was:

$$
\exists x, y \cdot \operatorname{geolink}(x, y) \wedge \operatorname{soclink}(x, y) \wedge \operatorname{Highlncome}(x) \wedge \operatorname{Highlncome}(y)
$$

$Q$ is still a minimal CQ: no atoms can be removed to get an equivalent query, even taking into account the integrity constraints. Thus Theorem 5.5 tells us that the canonical d-view is still minimally informative among the CQ-based d-views.

Theorem 5.1 tells us that the minimally informative d-views are among all views are given by the $\Sigma$ shuffle-invariant views. We note that there is one non-trivial shuffle that is invariant with respect to the constraint: the shuffle $\mu_{0}$ on the geolink source which swaps $x$ and $y$. One can compute directly from the definition that $\mu_{0}$ is invariant.

If we compute the shuffle views for the HighIncome source, we see that they are essentially the same as the canonical views. For example, for the type with $x \neq y$ we get the view

$$
(\operatorname{Highlncome}(x) \wedge \operatorname{HighIncome}(y)) \vee(\operatorname{Highlncome}(y) \wedge \operatorname{Highlncome}(x))
$$

but this is clearly the same as Highlncome $(x) \wedge \operatorname{Highlncome}(y)$. But if we compute the shuffle views for the soclink source, we see that the impact of $\mu_{0}$ is to give the invariant shuffle view:

$$
\operatorname{soclink}(x, y) \vee \operatorname{soclink}(y, x)
$$

Thus the minimally informative d-view in the presence of this constraint requires a disjunction on the soclink source, matching exactly the intuition we proposed in Example 1.5.

Injective shuffles and CQ Views. We have seen that the canonical d-view may be less informative than the shuffle invariant views. However, the canonical d-view can still be minimal for UN-disclosure even when it is not minimally informative. This was the situation in Example 1.5 in the presence of constraints: by using the power of disjunction in a shuffle, we could hide information, but we could not hide whether a Boolean CQ held. The key in this example was that the shuffles were injective: they never map multiple variables to the same variable. One can contrast this to the situation in Example 4.14. The utility query in that example admits a non-injective shuffle, identifying $x_{1}$ and $x_{2}$. And in that example the canonical views were not minimal even in terms of UN-disclosure of CQs. We prove that this phenomenon occurs generally:

THEOREM 5.9. Suppose every invariant shuffle of $Q$ is injective, and that our constraints consist of TGDs. Then the canonical views of $Q$ are UN-disclosure minimal 
PROOF. By Theorem 4.10, the shuffle invariant d-view returns minimal information, and in particular is minimal for $\mathrm{UN}$-disclosure.

We show that if a BC $p$ is UN-disclosed by the canonical d-view then it is UNdisclosed by the invariant shuffle d-view.

We know from Theorem 2.10 that $p$ must be UN-disclosed on the critical instance $\mathcal{I}_{0}$. That is, every instance $\mathcal{I}^{\prime}$ that agrees with $\mathcal{I}_{0}$ on the canonical d-view must satisfy $p$. Now suppose $\mathcal{I}^{\prime}$ agrees with $\mathcal{I}_{0}$ on the invariant shuffle views. Because the shuffles are all injective, each invariant shuffle view is safe, and on $\mathcal{I}_{0}$ returns either empty (for the types that impose some inequalities), or only tuples containing $*$. Then $\mathcal{I}^{\prime}$ must agree with $\mathcal{I}_{0}$ on the invariant shuffle views, since when all shuffles are injective, the invariant shuffle views without inequalities are just disjunctions of permutations of the canonical view. Thus the former returns a tuple of $*$ exactly when the latter does. But $p$ is then true on $\mathcal{I}^{\prime}$, which shows that it is $\mathrm{UN}$-disclosed by the invariant shuffle views.

\section{NON-LOCAL BACKGROUND KNOWLEDGE}

The simplest kind of non-local constraint is the replication of a table between sources. A replication constraint is a constraint of the form

$$
\forall \mathbf{x} . R_{1}(\mathbf{x}) \leftrightarrow R_{2}(\mathbf{x})
$$

where $R_{1}$ is a relation name associated to source $\mathrm{s}_{1}$ and $R_{2}$ is a relation name associated to a different source $s_{2}$. We will sometimes abuse notation by using a single relation name, say $R$, and express the same constraint as above by saying " $R$ is replicated between $\mathrm{s}_{1}$ and $\mathrm{s}_{2}$ " or " $R$ is shared between $\mathrm{s}_{1}$ and $\mathrm{s}_{2}$ ".

Unlike local constraints, these require some communication among the sources to enforce. Thus we can consider a replication constraint to be a restricted form of source-to-source communication.

\subsection{From minimally informative to UN non-disclosure minimality}

We will see that several new phenomena arise in the presence of replication constraints. Recall that with only local constraints, we have useful d-views with minimal information. We cannot guarantee the existence of such a d-view in the presence of replication:

THEOREM 6.1. There is a schema with a replication constraint $\Sigma$ and a BCQQ where there is no minimally informative useful d-view for $Q$ within the class of all views with respect to $\Sigma$.

Our proof of Theorem 6.1 uses a schema with unary relation names $R, S, T$. There are two sources: $R$ and $T$ are in different sources, and $S$ is replicated between the two sources. Let $Q$ be $\exists x \cdot R(x) \wedge S(x) \wedge T(x)$.

We will explain how our views act when the active domain of our instances is over the integers. The proof will easily be seen to extend to arbitrary instances (e.g., by having the views reveal all information outside of the integers).

Consider the function $F_{1}(x)=2 x+3$ for $x$ even and $2 x+2$ for $x$ odd, and also the

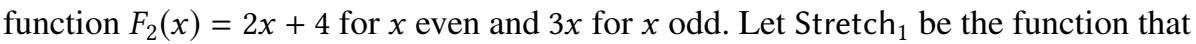
applies $F_{1}$ to the interpretation of a relation name, element by element. Similarly define Stretch ${ }_{2}$ using $F_{2}$. Notice that $F_{1}$ maps 0 to 3 and 1 to 4 , while $F_{2}$ maps 0 to 4 and 1 to 3 . Intuitively, these are functions that "stretch the instance" by distorting the values within 
the relations. For a number $i$ and any function $\mathcal{F}$ from instances to instance, such as Stretch or Stretch $_{2}$ above, and any instance $\mathcal{I}$, we let $(\mathcal{F})^{i}(\mathcal{I})$ be the instance resulting from applying $\mathcal{F} i$ times to $\mathcal{I}$.

We define a d-view $\mathcal{V}_{1}$ via an ECR, relating two instances $\mathcal{I}$ and $\mathcal{I}^{\prime}$ of the source exactly when $\mathcal{I}^{\prime}$ can be obtained by applying Stretch $h_{1}$ on $\mathcal{I}$ some number of times (applying it to both relation names of the source) or vice versa. That is, the ECR of $\mathcal{V}_{1}$ is the smallest equivalence relation containing each pair $(\mathcal{I}$, $\operatorname{Stretch}(\mathcal{I}))$. Let $\mathcal{V}_{2}$ be defined analogously using Stretch $\mathrm{h}_{2}$. Towards showing that $\mathcal{V}_{1}$ and $\mathcal{V}_{2}$ are useful we prove following claim, which captures their key properties.

CLAIM 3. If we have two d-instances satisfying the replication constraint, $\left(\mathcal{I}_{1}, \mathcal{I}_{2}\right)$ and $\left(\mathcal{I}_{1}^{\prime}, \mathcal{I}_{2}^{\prime}\right)$, with the instances of the replicated relation names non-empty, then:

- If $\mathcal{I}_{1}^{\prime}=\operatorname{Stretch}_{1}^{i}\left(\mathcal{I}_{1}\right), \mathcal{I}_{2}^{\prime}=\operatorname{Stretch}_{1}^{j}\left(\mathcal{I}_{2}\right)$ then $i=j$; and similarly for Stretch . $_{\text {. }}$

- If $\mathcal{I}_{1}^{\prime}=\operatorname{Stretch}_{1}^{i}\left(\mathcal{I}_{1}\right)$ and $\mathcal{I}_{2}=\operatorname{Stretch}_{1}^{j}\left(\mathcal{I}_{2}^{\prime}\right)$ then $i=j=0$; and similarly for Stretch .

Proof. Let $S$ be the content of the replicated relation name in $\left(\mathcal{I}_{1}, \mathcal{I}_{2}\right)$, while $S^{\prime}$ is the content of the replicated relation name in $\left(\mathcal{I}_{1}^{\prime}, \mathcal{I}_{2}^{\prime}\right)$.

We focus first on Stretch ${ }_{1}$. For the first item, let $c(S)=\min \{|x+2| \mid x \in S\}$. We can check directly that for any non-empty $S, c\left(\operatorname{Stretch}_{1}(S)\right)>c(S)$. Then $\operatorname{Stretch}_{1}^{i}(S)=$ $S^{\prime}=\operatorname{Stretch}_{1}^{j}(S)$ implies that $i=j$ as otherwise $c\left(\operatorname{Stretch}_{1}^{i}(S)\right)>c\left(\operatorname{Stretch}_{1}^{j}(S)\right)$ when $i>j$ and $c\left(\operatorname{Stretch}_{1}^{i}(S)\right)<c\left(\operatorname{Stretch}_{1}^{j}(S)\right)$ when $i<j$. For the second item we would have, $\operatorname{Stretch}_{1}^{i}(S)=S^{\prime}$ and $\operatorname{Stretch}_{1}^{j}\left(S^{\prime}\right)=S$ which means $\operatorname{Stretch}_{1}^{i+j}(S)=S$ which is only possible for $i+j=0$.

For Stretch ${ }_{2}$, the proof is the same, but now using the function $d$ defined as $d(S)=$ $\min \{|x+1.5| \mid x \in S\}$. We can check that $d(S)<d\left(\operatorname{Stretch}_{2}(S)\right)$ for any non-empty $S$.

From the claim, usefulness follows easily. Suppose we have $\left(\mathcal{I}_{1}, \mathcal{I}_{2}\right)$ satisfying $Q$, and $\left(\mathcal{I}_{1}^{\prime}, \mathcal{I}_{2}^{\prime}\right)$ is equivalent to $\left(\mathcal{I}_{1}, \mathcal{I}_{2}\right)$. Since $\left(\mathcal{I}_{1}, \mathcal{I}_{2}\right)$ satisfies $Q$, we know that the interpretation of the replicated relation name in $\mathcal{I}_{1}$ and $\mathcal{I}_{2}$ is non-empty, so the claim applies to tell us that $\mathcal{I}_{1}^{\prime}=\mathcal{I}_{1}, \mathcal{I}_{2}^{\prime}=\mathcal{I}_{2}$.

Now suppose $\mathcal{V}$ were a minimally informative useful d-view for $Q$. We must have $\mathcal{V}_{1}$ and $\mathcal{V}_{2}$ determine $\mathcal{V}$. Thus in particular if we have two local instances that agree on either $\mathcal{V}_{1}$ or $\mathcal{V}_{2}$, then they agree on $\mathcal{V}$.

Consider a d-instance $\mathcal{D}$ with $R=\{0\}, S=\{0,1\}, T=\{0\}$, and a d-instance $\mathcal{D}^{\prime}$ with $R=\{3\}, S=\{3,4\}, T=\{4\}$. These are both valid instances (i.e., the replication constraint is respected). But $\mathcal{D}^{\prime}$ is obtained from $\mathcal{D}$ by applying Stretch on $_{1}$ the source with $R$, and by applying Stretch $h_{2}$ on the other source. Thus $\mathcal{D}^{\prime}$ and $\mathcal{D}$ are indistinguishable by $\mathcal{V}$, but $Q$ has a match in $\mathcal{D}$ but not in $\mathcal{D}^{\prime}$. This contradicts the assumption that $\mathcal{V}$ is useful for $Q$. This completes the proof of Theorem 6.1.

Given Theorem 6.1, for the remainder of subsection we will focus on obtaining useful views that minimize the set of queries that are UN non-disclosed. We say that a d-view $\mathcal{V}$ is $U N$ non-disclosure minimal for $C Q Q$ within a class of views $C$ if: $\mathcal{V}$ is useful for $Q$ and for any BCQ $p$, if there is a d-view based on $C$ which is useful for $Q$ and UN non-disclosing for $p$, then $\mathcal{V}$ is $\mathrm{UN}$ non-disclosing for $p$. Proposition 2.5 implies that 
if $\mathcal{V}$ is minimally informative within $C$, then it is $\mathrm{UN}$ non-disclosure minimal for any BCQ $Q$ within $C$.

We can use the technique in the proof of Theorem 6.1 to show a more promising new phenomenon: there may be d-views that are useful for CQ $Q$ and $\mathrm{UN}$ non-disclosing for CQ $p$, but they are much more intricate than any query related to the canonical d-view of $Q$. In fact we can show that with a fully-replicated relation name in the utility query we can can get useful and UN non-disclosing views whenever this is not ruled out for trivial reasons:

THEOREM 6.2. [UN non-disclosure minimal d-views in the presence of replication] If $B C Q Q$ contains a relation name of non-zero arity replicated across all sources, then there is a d-view that is useful for $Q$ and $U N$ non-disclosing for BCQ $p$ if and only if there is no homomorphism of $p$ to $Q$. Further, we can use the same $d$-view for every such $p$ without a homomorphism into a given $Q$. In particular, there is a view that is $U N$ non-disclosure minimal for $Q$.

Thus even though we do not have minimally informative useful d-views, we have d-views that are optimal from the perspective of UN non-disclosure and utility for a fixed $Q$. Note that the condition on $p$ and $Q$ can be restated as saying that $Q$ does not entail $p$.

We now begin the proof of Theorem 6.2. For notational simplicity, we keep the replication constraints implicit, assuming that the replicated predicates are named $T$ in each source and $Q$ refers to this "global" $T$. One direction of the theorem is clear: if there is a homomorphism of $p$ to $Q$ and $V$ are useful for $Q$, then $V$ cannot be UN non-disclosing for $p$, since on any instance where $Q$ holds, the views will disclose $p$.

For the other direction, we show, as in the case without constraints, that there is a single d-view that works for any $p$ such that there is no homomorphism from $p$ to $Q$. We provide views that are not isomorphism-invariant, assuming that the active domain of instances is $\operatorname{Pair}(\mathcal{N})$ defined below.

We let $\mathcal{N}$ denote the natural numbers. $\operatorname{Pair}(\mathcal{N})$ is the set of terms built up from elements $\mathcal{N}$ by the pairing function: when $x$ and $y$ are in $\operatorname{Pair}(\mathcal{N})$, then so is $(x, y)$, the ordered pair consisting of $x$ and $y$. Note that all elements in $\operatorname{Pair}(\mathcal{N})$ have a finite height. We let height be the function taking such a term and returning the nesting depth of the pairing function. It is defined inductively as height $(x)=0$ for $x \in \mathcal{N}$, and $\operatorname{height}((x, y))=\max (\operatorname{height}(x)$, height $(y))+1$. Note in particular that all elements in $\operatorname{Pair}(\mathcal{N})$ have finite height.

Given two instances $\mathcal{I}_{1}$ and $\mathcal{I}_{2}$ for the same schema, the synchronous product of $\mathcal{I}_{1}$ and $\mathcal{I}_{2}$ is the instance defined as follows:

- elements of the instance are pairs $(x, y)$ with $x \in \mathcal{I}_{1}, y \in \mathcal{I}_{2}$;

- for each relation name $R$, we have $R\left(\left(x_{1}, y_{1}\right) \ldots\left(x_{n}, y_{n}\right)\right)$ holds exactly when $R\left(x_{1}, \ldots x_{n}\right)$ holds in $\mathcal{I}_{1}$ and $R\left(y_{1}, \ldots y_{n}\right)$ holds in $\mathcal{I}_{2}$.

Note that the projection on the first component is a homomorphism of the product to instance $\mathcal{I}_{1}$ and projection on the second component is a homomorphism to $\mathcal{I}_{2}$.

We consider the transformation Stretch on a d-instance that maps each local instance to its synchronous product with canondb $(Q)$. As the name implies, this transformation is very roughly analogous to Stretch ${ }_{1}$ and Stretch $_{2}$ from the proof of Theorem 6.1. In 
each case we are translating the concrete values in an instance, but leaving the relational structure in place.

Note that $\operatorname{Stretch}(\mathcal{D})$ is over the same schema as $\mathcal{D}$ and that $\operatorname{Stretch}(\mathcal{D})$ validates the replication constraint. Furthermore we suppose that the domain of canondb $(Q)$ is included in $\mathcal{N}$ which ensures that the minimal height of elements in the interpretation of the relation name $T$ of $\operatorname{Stretch}(\mathcal{D})$ will be the minimal height of elements in the interpretation of the relation name $T$ of $\mathcal{D}$ plus one.

Definition 6.3. We define $\equiv^{\mathrm{s}}$ on s-instances as the symmetric reflexive transitive closure of $\mathcal{R}$ defined as $\mathcal{I} \mathcal{R} \mathcal{I}^{\prime}$ when $\mathcal{I}=\operatorname{Stretch}\left(\mathcal{I}^{\prime}\right)$ for $\mathcal{I}, \mathcal{I}^{\prime}$ two s-instances.

Definition 6.4. We define $\equiv_{G}$ on d-instances as the symmetric reflexive transitive closure of $\mathcal{R}$ defined as $\mathcal{D} \mathcal{R} \mathcal{D}^{\prime}$ when $\mathcal{D}^{\prime}=\operatorname{Stretch}(\mathcal{D})$ for $\mathcal{D}, \mathcal{D}^{\prime}$ two d-instances.

Definition 6.5. The ECR $\equiv$ is defined as $\mathcal{D}_{1} \equiv \mathcal{D}_{2}$ exactly when for each source $\mathrm{s} \mathcal{D}_{1}^{\mathrm{s}} \equiv{ }^{\mathrm{s}} \mathcal{D}_{2}^{\mathrm{s}}$.

Proposition 6.6. For two d-instances $\mathcal{D}$ and $\mathcal{D}^{\prime}$ with $\mathcal{D} \vDash Q, \mathcal{D} \equiv \mathcal{D}^{\prime}$ if and only if $\mathcal{D} \equiv_{G} \mathcal{D}^{\prime}$.

Proof. Clearly $\mathcal{D} \equiv_{G} \mathcal{D}^{\prime}$ implies $\mathcal{D} \equiv \mathcal{D}^{\prime}$. Now let us suppose that $\mathcal{D} \equiv \mathcal{D}^{\prime}$ with $\mathcal{D} \vDash Q$ and let us show that $\mathcal{D} \equiv_{G} \mathcal{D}^{\prime}$.

Since $\mathcal{D}_{1} \equiv \mathcal{D}_{2}$, for each source $\mathrm{s}$, there exists $i$ such that $\mathcal{D}_{1}^{\mathrm{s}}=\operatorname{Stretch}\left(\mathcal{D}_{2}^{\mathrm{s}}\right)$ or $\mathcal{D}_{2}^{\mathrm{s}}=\operatorname{Stretch}^{i}\left(\mathcal{D}_{1}^{\mathrm{s}}\right)$, where the notation Stretch ${ }^{i}$ means iterating Stretch $i$ times. On a $\mathrm{d}$-instance $\mathcal{D}$ where the interpretation of the replicated relation name $T$ is not empty, the minimal height of elements appearing in the first position of $T$ needs to be equal for all sources $\mathrm{s}$. Therefore, if we have distinct sources $\mathrm{s}$ and $\mathrm{s}^{\prime}, i$ is a number witnessing the definition above for $\mathrm{s}$ and $i^{\prime}$ the number for $\mathrm{s}^{\prime}$ then we must have $i=i^{\prime}$. And we cannot have $\mathrm{s}$ and $\mathrm{s}^{\prime}$ such that $\mathcal{D}_{1}^{\mathrm{s}}=\operatorname{Stretch}^{i}\left(\mathcal{D}_{2}^{\mathrm{s}}\right)$ and $\mathcal{D}_{2}^{\mathrm{s}^{\prime}}=\operatorname{Stretch}^{i^{\prime}}\left(\mathcal{D}_{1}^{\mathrm{s}^{\prime}}\right)$ with $i, i^{\prime}>0$. Therefore, when the replicated predicate is not empty, $\mathcal{D} \equiv \mathcal{D}^{\prime}$ implies $\mathcal{D} \equiv_{G} \mathcal{D}^{\prime}$.

When $\mathcal{D} \vDash Q$ the replicated predicate $T$ is not empty (since it appears in $Q$ ) which proves that $\mathcal{D} \equiv_{G} \mathcal{D}^{\prime}$.

We now show that $\equiv$ is the view that we want.

PROPOSITION 6.7. The view corresponding to ECR $\equiv$ is useful for $Q$.

Proof. Let $\mathcal{D}$ and $\mathcal{D}^{\prime}$ be two d-instances and let us suppose $\mathcal{D} \equiv \mathcal{D}^{\prime}$ and $\mathcal{D} \vDash Q$. By Proposition 6.6, we have that $\mathcal{D} \equiv_{G} \mathcal{D}^{\prime}$. Recall that there is a homomorphism from $\operatorname{Stretch}(\mathcal{D})$ to $\mathcal{D}$. Thus it is clear that if $\operatorname{Stretch}(\mathcal{D}) \vDash=Q$ it must be that $\mathcal{D} \vDash Q$. On the other hand if we have any match $h$ of $Q$ in $\mathcal{D}$, we can extend it to a match of $Q$ in the product by taking any variable $x$ of $Q$ to $\left(h(x), c_{x}\right)$ where $c_{x}$ is the constant corresponding to $x$ in canondb $(Q)$. From $\mathcal{D} \equiv_{G} \mathcal{D}^{\prime}$ either $\mathcal{D}$ or $\mathcal{D}^{\prime}$ can be obtained from the other by applying Stretch, and so $\mathcal{D} \mid=Q$ implies $\mathcal{D}^{\prime} \mid=Q$.

PROPOSITION 6.8. The view corresponding to ECR $\equiv$ is UN non-disclosing for $p$.

ProOF. Given an instance $\mathcal{D}$, we know that $\operatorname{Stretch}(\mathcal{D})$ has a homomorphism into $\operatorname{canondb}(Q)$. Therefore there is no homomorphism from $p$ into $\operatorname{Stretch}(\mathcal{D})$ because if there were, we would have a homomorphism from $p$ into canondb $(Q)$, a contradiction of the assumption that $Q$ did not entail $p$. 
Putting together the results above we complete the proof of Theorem 6.2.

Example 6.9. We give an example of the power of Theorem 6.2, and we highlight the difference from the situation with only local constraints. Suppose we have two sources, with one binary relation name $S$ replicated between the two, and each source having one non-replicated binary relation name, $R$ in one source and $T$ in the other. The utility query $Q$ is $\exists x, y \cdot R(x, y) \wedge S(x, y) \wedge T(x, y)$ and the secret query $p$ is $\exists x \cdot R(x, x)$.

Since $p$ is not entailed by $Q$, Theorem 6.2 implies that there are views that are useful for $Q$ but $\mathrm{UN}$ non-disclosing for $p$. But it is easy to see that the canonical d-view of $Q$ is UN disclosing for $p$.

We show that for this particular example there do indeed exist relational algebra views that were useful for $Q$ and $\mathrm{UN}$ non-disclosing for $p$. Thus in exploiting replication we can sometimes stay within a standard class of views. We now explain how to use relational algebra views in this example.

Given an instance of the $R$ source $\mathcal{I}_{R}$, we say an $R$-harmless pair is any pair of elements $\left(x_{1}, x_{2}\right)$ of $\mathcal{I}_{R}$, where:

- $x_{1}$ and $x_{2}$ are connected by both $R$ and $S$ edges;

- there is no $S$ self-loop on $x_{1}$;

- $x_{2}$ has no outgoing $S$ edges, and $x_{2}$ is the unique element that is a target of an $S$ edge from $x_{1}$ with no outgoing $S$ edges.

The modification of such a pair $\left(x_{1}, x_{2}\right)$ is the pair $\left(x_{1}, x_{1}\right)$.

Our view on the $R$-source takes as input an instance $\mathcal{I}$ of the $R$ source, and returns all pairs that are modifications of $R$-harmless pairs, unioned with pairs that are in $S \cap R$ but are not $R$-harmless.

A $T$-harmless pair is a pair of elements in the $T$-source, defined similarly but replacing $R$ with $T$. A modification of such a pair is as above. Analogously, our view on the $T$ source returns all pairs that are modifications of $T$-harmless pairs, unioned with pairs that are in $S \cap T$ but are not harmless. It is clear that these views can be expressed in relational algebra.

We first show that the views are UN non-disclosing for $p$. Consider an instance $\mathcal{D}=(R, T, S)$ where $p$ holds, with $S$ the shared relation name. We will construct an instance $\mathcal{D}^{\prime}=\left(R^{\prime}, T^{\prime}, S^{\prime}\right)$ with the same view images, but where $p$ does not hold. We let $V_{R}(\mathcal{I})$ be the content for the view for the $R$-source on instance $\mathcal{I}$, and similarly $V_{T}(\mathcal{I})$.

We begin our construction of $\mathcal{D}^{\prime}$ by describing the interpretation of the shared relation name $S^{\prime}$. For each element $v$ in either view image $\left(V_{R}(\mathcal{D})\right.$ or $\left.V_{T}(\mathcal{D})\right)$, we create an $S$ edge from $v$ to a new element $n_{v}$. We also include all pairs in either view that are not self-loops.

We now turn to describing the content of $R^{\prime}$. It includes all edges in the view $V_{R}(\mathcal{D})$ that are not self-loops. It also contains an edge from $v$ to $n_{v}$ if $(v, v)$ is in $V_{R}(\mathcal{D})$. The content of the non-shared relation name $T^{\prime}$ is defined analogously.

It is clear that the new instance does not satisfy $p$. We need to show that it agrees with $\mathcal{D}$ on each view. Note that all of the pairs $\left(v, n_{v}\right)$ such that $(v, v)$ is in $V_{R}(\mathcal{D})$ are $R$-harmless. A pair of the form $(c, v)$ where $v$ is one of the original nodes of the instance, cannot be $R$-harmless, since $v$ has an outgoing $S$ edge. Pairs of the form $\left(c, n_{v}\right)$ where $c \neq v$ are not $R$-harmless because there is no $S$ or $R$ edge between them. Thus the $R$-harmless pairs are exactly those of the form $\left(v, n_{v}\right)$ where $(v, v) \in V_{R}(\mathcal{D})$. But 
the view will produce all such pairs $(v, v)$. We can conclude that $V_{R}(\mathcal{D})$ and $V_{R}\left(\mathcal{D}^{\prime}\right)$ agree on pairs of the form $(v, v)$. The next case to consider consists of pairs in the view $V_{R}(\mathcal{D})$ of the form $(c, d)$ with $d \neq c$. By definition, such pairs are included in both $S^{\prime}$ and $R^{\prime}$. They are not $R$-harmless in $\mathcal{D}^{\prime}$, since $d$ has an outgoing edge to $n_{d}$. Thus they are included in $V_{R}\left(\mathcal{D}^{\prime}\right)$. Conversely, if a pair $(c, d)$ with $c \neq d$ does not occur in $V_{R}(\mathcal{D})$, we can argue that it is not in $V_{R}\left(\mathcal{D}^{\prime}\right)$. This follows since we will not have $R^{\prime}$ hold of $(c, d)$. We have thus shown that the new instance agrees with $\mathcal{D}$ on each view.

We next show that the views are useful for $Q$, by arguing that the BCQ $Q$ is equivalent to the non-emptiness of the intersection of $V_{R}$ and $V_{T}$.

In one direction, we suppose $Q$ has a match $(c, d)$ in an instance $\mathcal{D}$, and we argue that $(c, d)$ is in the intersection of $V_{R}(\mathcal{D})$ and $V_{T}(\mathcal{D})$. Note that such a pair is $R$-harmless if and only if it is $T$-harmless because $T \wedge R$ holds of it in $\mathcal{D}$. Thus we will distinguish pairs that are harmless (meaning $R$ or $T$-harmless) versus pairs that are not harmless. If $(c, d)$ is a harmless pair, then $(c, c)$ will be in the intersection of $V_{R}$ and $V_{T}$ within $\mathcal{D}$. While if $(c, d)$ is not a harmless pair, then $(c, d)$ will be in the intersection of $V_{R}$ and $V_{T}$.

We now suppose that $V_{R}(\mathcal{D})$ and $V_{T}(\mathcal{D})$ intersect, and show that $Q$ must hold on $\mathcal{D}$. First, suppose that the intersection has a pair $(c, d)$ with $d \neq c$. Then it is clear that $(c, d)$ must be a match of $Q$. The more interesting case is when there is an element in the intersection of the form $(c, c)$. As a first subcase, suppose $S(c, c)$ holds within $\mathcal{D}$. Then there are no $R$-harmless or $T$-harmless pairs of the form $(c, d)$ and thus $(c, c)$ could not have been produced in either $V_{R}$ or $V_{T}$ as a modification. Hence $(c, c)$ must have gotten into $V_{R}(\mathcal{D})$ because $\mathcal{D} \mid=S(c, c) \wedge R(c, c)$, while $(c, c)$ was in view $V_{T}$ because $\mathcal{D} \mid=S(c, c) \wedge T(c, c)$. Thus we have a match of $Q$. The second subcase is where $(c, c)$ does not hold in $S$ within $\mathcal{D}$. Then $(c, c)$ must have been produced as a modification of an $R$-harmless pair $(c, d)$ and as a modification of some $T$-harmless pair $\left(c, d^{\prime}\right)$. But from the uniqueness condition in $R$-harmlessness and $T$-harmlessness, we conclude that $d=d^{\prime}$. Now $(c, d)$ is a match of $Q$.

\subsection{Negative results on UN non-disclosure minimality}

Theorem 6.2 shows that we can still find UN non-disclosure minimal d-views even in the presence of replication. We now proceed to show some restrictions on what one can achieve even in terms of this more restricted notion of "minimally expressive" d-view.

Separating the power of general and isomorphism-invariant d-views. The views used in Theorem 6.2 are not isomorphism-invariant: like the views from Theorem 6.1, the product construction can be seen as applying some value transformation on the elements of each instance. We can show - in sharp contrast to the situation with only local constraints - that with replication, even to achieve this weaker notion of minimality, it may be essential to use d-views based on queries that are not isomorphisminvariant.

THEOREM 6.10. There is a d-schema with a replication constraint, along with $B C Q s Q$ and $p$ such that there is a d-view useful for $Q$ and UN non-disclosing for $p$, but there is no such d-view based on queries returning values in the active domain and commuting with isomorphisms. In particular, we cannot find a UN non-disclosure minimal d-view that is isomorphism-invariant in the above sense. 
For a query $Q_{V}$ that always returns tuples containing only values in the active domain of the input, we will use the following restricted version of the isomorphism-invariance property:

If $\mathcal{I}$ and $\mathcal{I}^{\prime}$ are source instances for $Q_{V}$ with $\mathcal{I}^{\prime}$ formed from $\mathcal{I}$ via an isomorphism that is the identity on adom $(V(I))$, then $\mathcal{I}$ and $h(\mathcal{I})$ agree on $Q_{V}$.

For example, any query in relational algebra has the isomorphism-invariance property above. We now proceed with the proof of Theorem 6.10. Consider the following Boolean CQs (existentially quantifiers omitted):

$$
\begin{aligned}
Q & =P(x) \wedge P(y) \wedge S(y) \wedge S(z) \wedge T(z) \wedge T(x) \wedge R(w) \\
p & =S(x) \wedge T(x) \wedge P(x)
\end{aligned}
$$

It is easy to see that $Q$ does not entail $p$. Since there is a replicated relation name mentioned in the query, Theorem 6.2 implies that we can get a d-view that is useful for $Q$ and UN non-disclosing for $p$.

Now, by way of contradiction, fix a d-view $\mathcal{V}$ that is useful for $Q$, returns only facts whose values lie in the active domain, and where the queries in the view definitions satisfy the isomorphism-invariance property. Our proof will involve two auxiliary propositions about this setting.

Proposition 6.11. Suppose d-view $\mathcal{V}$ is useful for $Q$ and satisfies the active domain and isomorphism-invariance properties above. Given two instances $\mathcal{I}_{1}$ and $\mathcal{I}_{2}$ for the source whose unreplicated relation name is $G$, if $\mathcal{I}_{1}$ and $\mathcal{I}_{2}$ agree on the replicated relation name and agree on each view in $\mathcal{V}$ for this source, then they must agree on $G$.

PROOF. Let $c \in G\left(\mathcal{I}_{1}\right)$ and form $\mathcal{D}_{1}$ by choosing a context for the other unshared relation names with the interpretation of each relation name containing only c. Let $\mathcal{D}_{2}$ be formed similarly from $\mathcal{I}_{2}$. Then $\mathcal{D}_{1}$ and $\mathcal{D}_{2}$ agree on all views in $\mathcal{V}$. There is a match of $Q$ on $\mathcal{D}_{1}$ so the same must be true of $\mathcal{D}_{2}$, since $\mathcal{V}$ is useful for $Q$. Clearly the witness can only be $c$, thus $c \in G\left(\mathcal{I}_{2}\right)$.

In our next proposition, we let $\mathcal{D}_{0}$ be the critical instance, recalling that this has only a single element $*$ with every relation name holding of it. We show that $\mathcal{D}_{0}$ contradicts that $\mathcal{V}$ is UN non-disclosing for $p$. Note that adom $\left(\mathcal{V}_{0}\right) \subseteq\{*\}$ since we assume views return facts containing only elements in the active domain of the input instance.

PROPOSITION 6.12. Let $\mathcal{V}$ be views that are useful for $Q$, satisfying isomorphisminvariance property and returning only elements in the active domain. Let $\mathcal{D}$ agree with $\mathcal{D}_{0}$ on $\mathcal{V}$. For any unreplicated relation name $G$, we have $\mathcal{D} \mid=\forall x((G(x) \wedge x \neq *) \rightarrow$ $R(x))$.

Proof. Let $\mathcal{I}$ be the restriction of $\mathcal{D}$ to the $G$ source, and suppose $G(v)$ holds in $\mathcal{I}$ for $v \neq *$. Let $c$ be a fresh value and $\mathcal{I}^{\prime}$ be the result of applying an isomorphism swapping $v$ and $c$. Since $v \neq *$, the isomorphism-invariance property implies that $\mathcal{I}$ and $\mathcal{I}^{\prime}$ agree on the views. Because $\mathcal{I}^{\prime}$ and $\mathcal{I}$ disagree on $G$, Proposition 6.11 implies they cannot agree on the replicated relation name. Note that $c$ was fresh (hence in particular was not in the interpretation of $R$ within $\mathcal{I}$ ), and no other change occurred in $R$ outside of the swap of $v$ and $c$. Thus the only way that $I$ and $I^{\prime}$ can disagree on $R$ is because $R(v)$ held in $\mathcal{I}$. 
With these propositions in hand we are ready to complete the argument for Theorem 6.10. By usefulness of $\mathcal{V}$ and the fact that $\mathcal{D}_{0}$ has a match of $Q$, we know that $\mathcal{D}$ has a match of $Q$ with $x_{0}, y_{0}, z_{0}, w_{0}$. We first consider the case where two of $x_{0}, y_{0}, z_{0}$ are the same. In this case $p$ has a match.

Now suppose the match has all of $x_{0}, y_{0}, z_{0}$ distinct. At most one of them can be *, so assume $y_{0}$ and $z_{0}$ are not $*$. Proposition 6.12 implies that they are both in $R$. Since $y_{0}$ and $z_{0}$ are not in adom $\left(\mathcal{V}_{0}\right)$, when we consider the result of swapping $y_{0}$ and $z_{0}$, it does not impact the views, by the isomorphism-invariance property. Since this swap also does not change $R$, we can apply Proposition 6.11 to conclude that all local sources agree on $y_{0}$ and $z_{0}$. In particular we have $P\left(y_{0}\right) \wedge S\left(z_{0}\right)$, thus we have $P\left(z_{0}\right) \wedge S\left(y_{0}\right)$. But then either $y_{0}$ or $z_{0}$ gives a match of $p$.

This completes the proof of Theorem 6.10.

Separating the power of Relational Algebra-based d-views and DCQ-based dviews. In Theorem 6.10 we have seen that the UN non-disclosure minimal d-view may not be isomorphism-invariant. In particular, the theorem shows we may be able to find a $\mathrm{d}$-view that balances supporting the querying of a CQ $Q$ while avoiding UN disclosure of CQ $p$, but we may not be able to do it with an isomorphism-invariant d-view. We will now give another separation result, showing that we may be able to design a d-view that is useful for $Q$ and $\mathrm{UN}$ non-disclosing for $p$ based on relational algebra, while not being able to construct such a d-view with DCQ views. This contrasts again with the situation without replication, where the minimal information, and hence the minimal non-disclosure, can always be achieved by DCQ-based views.

We will prove a more general result. Instance $\mathcal{I}_{1}$ is a subinstance of $\mathcal{I}_{2}$ if $\mathcal{I}_{1}$ is a subset of $\mathcal{I}_{2}$ when they are seen as sets of facts. In other words, for every relation name, its interpretation in $\mathcal{I}_{1}$ is a subset of its interpretation in $\mathcal{I}_{2}$. A view $V$ is monotone if the output whenever $\mathcal{I}_{1}$ is a subinstance of $\mathcal{I}_{2}$, the output of $\mathrm{V}$ on $\mathcal{I}_{1}$ is a subset of the output on $\mathcal{I}_{2}$. A set of views $\mathcal{V}$ is monotone if each view in the set is monotone. Note that UCQs, DCQs, along with their extensions with inequalities, are all monotone. There are a number of powerful query languages that extend UCQs, such as Datalog [1], that also define only monotone queries.

THEOREM 6.13. There is a d-schema with replication constraints along with BCQs $Q$ and $p$, such that there is a relational algebra based $d$-view that is useful for $Q$ and UN non-disclosing for $p$, but none based on monotone views.

Proof. We use the d-schema $Q$, and $p$ from Example 6.9. We have already shown that there is a relational algebra based d-view that is useful for $Q$ and $\mathrm{UN}$ non-disclosing for $p$, so we focus on showing that this cannot be done with monotone views.

Say that an element $d$ appears non-trivially in a $k$-ary relation $S$ if there is a tuple $\mathbf{t}$ in $S$ with $t_{i}=d$, a $\mathbf{t}^{\prime}$ formed from $\mathbf{t}$ by setting $t_{i}^{\prime}$ to $d^{\prime} \neq d$ while $t_{j}=t_{i}$ for $j \neq i$, such that $\mathbf{t}^{\prime}$ is not in $S$. For the output of a CQ or UCQ view, there is no difference between appearing non-trivially and being in the active domain of the view output. But for unsafe views there is a difference, since it is possible that every element appears in the output, but if $d$ appears non-trivially in the output of a DCQ then there must be some disjunct in the DCQ such that $d$ satisfies the disjunct.

For elements $c$ and $d$ let $\mathcal{I}_{c, d}$ be the instance where each relation name is interpreted by the single pair $(c, d)$. We first claim that on this instance each of $c, d$ needs to be 
appear non-trivially in the output of some view. We show the claim for $d$, with the claim for $c$ being symmetric.

Suppose not, and fix $e$ distinct from both $c$ and $d$. Consider $\mathcal{I}_{0}=\mathcal{I}_{c, d} \cup \mathcal{I}_{c, e}$ along with $\mathcal{I}_{1}$ the instance with only $(c, d)$ in $R$, both $(c, d)$ and $(c, e)$ in $S$ and in $S$ but only $(c, e) \in T$. If $d$ does not occur non-trivially in the view output in $\mathcal{I}_{c, d}$, the views must return the same result on $\mathcal{I}_{c, e}$ and $\mathcal{I}_{c, d}$. Now $\mathcal{I}_{1}$ is a subinstance of $\mathcal{I}_{c, d}$ on the $R$ source, and a subinstance of $\mathcal{I}_{c, e}$ on the $S$ source. Thus by monotonicity of the views, the output of each view on $\mathcal{I}_{1}$ is contained in the corresponding output on $\mathcal{I}_{0}$. On the other hand, since $\mathcal{I}_{0}$ is a subinstance of $\mathcal{I}_{1}$, the view outputs must be identical on $\mathcal{I}_{1}$ and $\mathcal{I}_{0}$. But since $Q$ holds on $\mathcal{I}_{0}$ and not on $\mathcal{I}_{1}$, this contradicts usefulness of the views.

Now consider an instance of the form $\mathcal{I}_{c, c}$ for an element $c$. The secret query $p$ holds, and $Q$ holds. So by UN non-disclosure there must be an instance $\mathcal{I}^{\prime}$ with the same view image as $\mathcal{I}_{c, c}$ where $p$ fails and $Q$ holds. Since $Q$ holds, $\mathcal{I}^{\prime}$ must contain $\mathcal{I}_{e, f}$ for some $e \neq f$. By the assertions above, there must be a view where $e$ appears non-trivially in its output and also a view where $f$ appears non-trivially. Clearly, for each view output on $\mathcal{I}_{c, c}$, only $c$ can appear non-trivially. Since one of $e, f$ must be distinct from $c$, this is a contradiction of the fact that $\mathcal{I}_{c, c}$ and $\mathcal{I}^{\prime}$ must agree on the views.

This completes the proof of Theorem 6.13.

Lack of UN disclosure minimality d-views when restricting to CQ views. We now turn to another limitation of Theorem 6.2. It states that there are d-views minimal for UN disclosure even in the presence of replication. But it tells us nothing about whether there are d-views that are minimal for UN disclosure in the presence of replication within the class of CQ-based d-views. Unfortunately, there we can get quite strong negative results.

THEOREM 6.14. There is a schema with a replication constraint and a utility query $Q$ such that there is no CQ-based d-view $\mathcal{V}$ that is minimal for UN non-disclosure within the class of $C Q$ views. In particular, there is no minimally informative useful $d$-view for $Q$ within the class of $C Q$-based views.

Note that this does not follow from Theorem 6.10, which only concerns UN nondisclosure minimality within the class of all views.

We will prove a stronger statement, along the lines of what is shown in Subsection 4.2. Recall from there that a query $Q$ is homomorphism-invariant when, for all pairs of instances $\mathcal{I}$ and $\mathcal{I}^{\prime}$ and all homomorphisms $\mu$ from $\mathcal{I}$ to $I^{\prime}$ then $\mu(Q(\mathcal{I})) \subseteq Q\left(\mathcal{I}^{\prime}\right)$ and adom-based when adom $(Q(\mathcal{I})) \subseteq$ adom $(I)$. We will show that views based on such queries cannot be minimally informative.

Our schema has two sources, $\mathcal{P}$ and $\mathcal{S} . \mathcal{P}$ contains a binary relation name $P$ and $\mathcal{S}$ contains a binary relation $S$. Both sources contain a shared binary relation name $T$. The utility query is $Q=\exists w, x, y, z \cdot T(x, y) \wedge S(y, z) \wedge T(z, w) \wedge P(w, x)$. It is illustrated in Figure 2.

We consider three secrets:

- $p_{1}=\exists x . S(x, x)$,

- $p_{2}=\exists x, y \cdot T(x, y) \wedge S(y, x)$,

- $p_{3}=\exists x, y, z \cdot T(x, y) \wedge S(y, z) \wedge T(z, x)$.

It is easy to see that, for each of these secrets, there exists a d-view based on CQs that is useful for $Q$ and $\mathrm{UN}$ non-disclosing for the secret. Indeed, we have: 


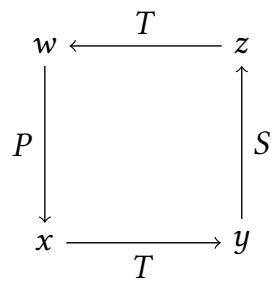

Fig. 2. Query $Q$ counter-example for theorem 6.14

- $Q_{\mathcal{S}}(x, w)=\exists y, z \cdot T(x, y) \wedge S(y, z) \wedge T(z, w)$ and $Q_{\mathcal{P}}(w, x)=P(w, x)$ for $p_{1}$,

- $Q_{\mathcal{S}}(y, w)=\exists z \cdot S(y, z) \wedge T(z, w)$ and $Q_{\mathcal{P}}(w, y)=\exists y \cdot P(w, x) \wedge T(x, y)$ for $p_{2}$

- $Q_{\mathcal{S}}(y, z)=S(y, z)$ and $Q_{\mathcal{P}}(y, z)=\exists w, x \cdot T(y, w) \wedge P(w, x) \wedge T(x, y)$ for $p_{3}$.

Now consider a d-view $\mathcal{V}$, useful for $Q$, homomorphism-invariant, and adom-based. We will show that $\mathcal{V}$ is necessarily $\mathrm{UN}$ disclosing for one of the secrets among $p_{1}, p_{2}, p_{3}$. We now claim that:

LEMma 6.15. Any $d$-view $\mathcal{V}$ that is useful for $Q$, homomorphism-invariant, and adom-based must necessarily be UN disclosing for one of the secrets among $p_{1}, p_{2}, p_{3}$.

The remainder of this subsection is devoted to the proof of this lemma, which completes the theorem. The proof will follow the technique used in Proposition 4.15.

Let us consider the following d-instance $\mathcal{D}_{Q}$ shown in Figure 3. This is nothing more than an isomorphic copy of canondb $(Q)$. Recall that $\mathcal{V}^{\mathcal{S}}$ denotes the component of the d-view $\mathcal{V}$ for source $\mathcal{S}$, and thus $\mathcal{V}^{\mathcal{S}}\left(\mathcal{D}_{Q}\right)$ denotes the view image of the instance $\mathcal{D}_{Q}$ under this view.

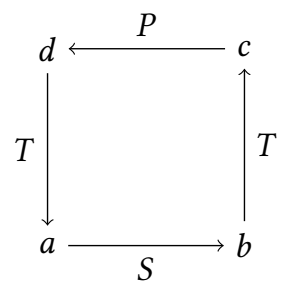

Fig. 3. Instance $\mathcal{D}_{Q}$

CLAIM 4. $\operatorname{adom}\left(\mathcal{V}^{\mathcal{S}}\left(\mathcal{D}_{Q}\right)\right)$ includes either a or $d$.

Proof. Let us consider the instance $\mathcal{I}_{1}$ depicted in Figure 4, and let us show that

(1) adom $\left(\mathcal{V}^{\mathcal{S}}\left(\mathcal{I}_{1}\right)\right)$ includes $a$ or $d$ if and only if $\operatorname{adom}\left(\mathcal{V}^{\mathcal{S}}(\mathcal{D})\right)$ includes $a$ or $d$, and

(2) $\operatorname{adom}\left(\mathcal{V}^{\mathcal{S}}\left(\mathcal{I}_{1}\right)\right)$ includes either $a$ or $d$.

For the first item since $\mathcal{D}_{Q} \subseteq \mathcal{I}_{1}$ we know that $\operatorname{adom}\left(\mathcal{V}^{\mathcal{S}}\left(\mathcal{D}_{Q}\right)\right) \subseteq \operatorname{adom}\left(\mathcal{V}^{\mathcal{S}}\left(\mathcal{I}_{1}\right)\right)$. Consider the homomorphism $\mu$ that is the identity on the elements $a, b, c$ and $d$ but sends $f$ to $d, e$ to $a, h$ to $c$, and $g$ to $b$.

The image of $\mathcal{I}_{1}$ is $\mathcal{D}_{Q}$ and, since $\mu\left(\mathcal{I}_{1}\right)=\mathcal{D}_{Q}$, we know from homomorphisminvariance that $\mu\left(\mathcal{V}^{\mathcal{S}}\left(\mathcal{I}_{1}\right)\right) \subseteq \mathcal{V}^{\mathcal{S}}\left(\mathcal{D}_{Q}\right)$. If $\operatorname{adom}\left(\mu\left(\mathcal{V}^{\mathcal{S}}\left(\mathcal{I}_{1}\right)\right)\right)$ contains $a$ or $d$, $\operatorname{adom}\left(\mu\left(\mathcal{V}^{\mathcal{S}}\left(\mathcal{D}_{Q}\right)\right)\right)$ 

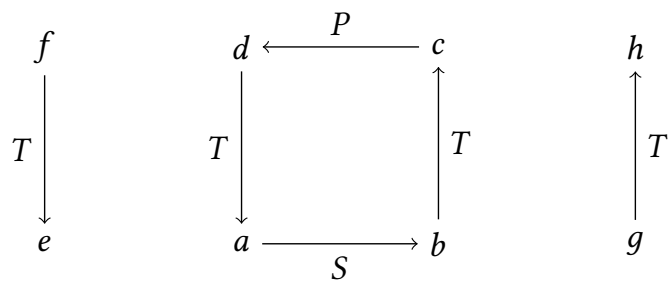

Fig. 4. Instance $\mathcal{I}_{1}$

contains $a$ or $d$. Note that this also proves that when $e$ or $f$ in $\operatorname{adom}\left(\mu\left(\mathcal{V}^{\mathcal{S}}\left(\mathcal{I}_{1}\right)\right)\right), a$ or $d$ are in $\operatorname{adom}\left(\mu\left(\mathcal{V}^{\mathcal{S}}\left(\mathcal{I}_{1}\right)\right)\right)$.

To prove that adom $\left(\mathcal{V}^{\mathcal{S}}\left(\mathcal{I}_{1}\right)\right)$ includes $a$ or $d$, let us consider the instance $\mathcal{I}_{2}$, depicted in Figure 5. This instance is obtained from $\mathcal{I}_{1}$ by replacing the $\mathcal{S}$ source with its image through the isomorphism $v$ that exchanges $e$ and $a$ and $f$ and $d$.

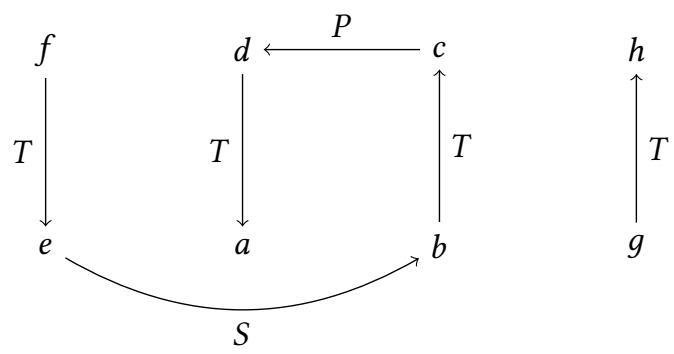

Fig. 5. Instance $\mathcal{I}_{2}$

If $\{a, d\} \cap \operatorname{adom}\left(\mathcal{V}^{\mathcal{S}}\left(\mathcal{I}_{1}\right)\right)=\emptyset$, then $\{f, e\} \cap \operatorname{adom}\left(\mathcal{V}^{\mathcal{S}}\left(\mathcal{I}_{1}\right)\right)=\emptyset$. And if $\{a, d, e, f\} \cap$ adom $\left(\mathcal{V}^{\mathcal{S}}\left(\mathcal{I}_{1}\right)\right)=\emptyset$, then $\mathcal{V}^{\mathcal{S}}\left(\mathcal{I}_{1}\right)=v\left(\mathcal{V}^{\mathcal{S}}\left(\mathcal{I}_{1}\right)\right)$. Since $v$ is an isomorphism between $\mathcal{I}_{1}^{\mathcal{S}}$ and $\mathcal{I}_{2}^{\mathcal{S}}, \mathcal{V}^{\mathcal{S}}\left(\mathcal{I}_{1}\right)=v\left(\mathcal{V}^{\mathcal{S}}\left(\mathcal{I}_{1}\right)\right)=\mathcal{V}^{\mathcal{S}}\left(v\left(\mathcal{I}_{1}\right)\right)=\mathcal{V}^{\mathcal{S}}\left(\mathcal{I}_{2}\right)$. And as the $\mathcal{P}$ sources are identical we conclude that $\mathcal{V}\left(\mathcal{I}_{1}\right)=\mathcal{V}\left(\mathcal{I}_{2}\right)$. This implies that $\mathcal{V}$ is not useful for $Q$ as $Q \vDash \mathcal{I}_{1}$ but $Q \not \models \mathcal{I}_{2}$, a contradiction.

ClAim 5. adom $\left(\mathcal{V}^{\mathcal{S}}\left(\mathcal{D}_{Q}\right)\right)$ contains either b or $c$.

PROOF. Same proof as above (by symmetry).

Claim 6. $\mathcal{V}$ is $U N$-disclosing:

(1) for $p_{1}$ when $\{a, b\} \subseteq \operatorname{adom}\left(\mathcal{V}^{\mathcal{S}}\left(\mathcal{D}_{Q}\right)\right)$;

(2) for $p_{2}$ when $\{d, b\} \subseteq \operatorname{adom}\left(\mathcal{V}^{\mathcal{S}}\left(\mathcal{D}_{Q}\right)\right)$;

(3) for $p_{2}$ when $\{a, c\} \subseteq \operatorname{adom}\left(\mathcal{V}^{\mathcal{S}}\left(\mathcal{D}_{Q}\right)\right)$;

(4) for $p_{3}$ when $\{d, c\} \subseteq \operatorname{adom}\left(\mathcal{V}^{\mathcal{S}}\left(\mathcal{D}_{Q}\right)\right)$.

Proof. Consider the critical d-instance, $\mathcal{I}=\{T(*, *), S(*, *), P(*, *)\}$, and take a d-instance $\mathcal{E}$ such that $\mathcal{V}(\mathcal{E})=\mathcal{V}(\mathcal{I})$. Since $\mathcal{V}$ is useful for $Q$, there exists $x, y, z, w$ such that $Q=T(x, y) \wedge S(y, z) \wedge T(z, w) \wedge P(w, x)$ holds in $\mathcal{E}$ (the $x, y, z$ and $w$ being not necessarily different). 
Let $\xi$ be the homomorphism from $\mathcal{D}_{Q}$ to $\mathcal{E}$ that maps $d$ to $x, a$ to $y, b$ to $z$, and $c$ to w. $\xi$ maps $\mathcal{D}_{Q}$ to a subset of $\mathcal{E}$ and this proves that $\xi\left(\mathcal{V}^{\mathcal{S}}\left(\mathcal{D}_{Q}\right)\right) \subseteq \mathcal{V}^{\mathcal{S}}(\mathcal{E})=\mathcal{V}^{\mathcal{S}}(\mathcal{I})$. But adom $\left(\mathcal{V}^{\mathcal{S}}(\mathcal{I})\right)=\{*\}$. We now do a case analysis, depending on the four cases of the claim.

- Suppose $\{a, b\} \subseteq \operatorname{adom}\left(\mathcal{V}^{\mathcal{S}}\left(\mathcal{D}_{Q}\right)\right)$. By homomorphism invariance, $\{\xi(a), \xi(b)\} \subseteq$ $\operatorname{adom}\left(\mathcal{V}^{\mathcal{S}}\left(\xi\left(\mathcal{D}_{Q}\right)\right)\right)=\operatorname{adom}\left(\mathcal{V}^{\mathcal{S}}(\mathcal{I})\right)=\{*\}$, and thus $y=\xi(a)=*=\xi(b)=z$ which means that $p_{1}$ holds in $\mathcal{E}$.

- Suppose $\{d, b\} \subseteq \operatorname{adom}\left(\mathcal{V}^{\mathcal{S}}\left(\mathcal{D}_{Q}\right)\right)$. Then $x=\xi(d)=\xi(b)=z$, and thus $p_{2}$ holds in $\mathcal{E}$.

- Suppose $\{a, c\} \subseteq \operatorname{adom}\left(\mathcal{V}^{\mathcal{S}}\left(\mathcal{D}_{Q}\right)\right)$. Then $y=\xi(a)=\xi(c)=w$, and thus $p_{2}$ holds in $\mathcal{E}$.

- Suppose $\{d, c\} \subseteq \operatorname{adom}\left(\mathcal{V}^{\mathcal{S}}\left(\mathcal{D}_{Q}\right)\right)$. Then $x=\xi(d)=\xi(c)=w$, and thus $p_{3}$ holds in $\mathcal{E}$

Since $\mathcal{E}$ was an arbitrary instance agreeing with the views, we have shown that $\mathcal{V}$ is $\mathrm{UN}$-disclosing for one of the secrets $p_{1}, p_{2}, p_{3}$.

By combining claims 4, 5, and 6, we get the proof of Lemma 6.15.

Theorem 6.14 follows easily from Lemma 6.15. Suppose there were a set of views $\mathcal{V}$ in the class that were minimal for $\mathrm{UN}$ non-disclosure. Since for each $i \in\{1,2,3\}$, there are d-views based on CQs that are useful for $Q$ and non-disclosing for $p_{i}, \mathcal{V}$ could not disclose any of $p_{1}, p_{2}, p_{3}$. But this contradicts Lemma 6.15 .

\section{DISCUSSION AND OUTLOOK}

We have studied the ability to design views that satisfy diverse goals: expressiveness requirements in terms of full disclosure of a specified set of queries in the context of data integration, and inexpressiveness restrictions in terms of either minimal utility or minimizing disclosure of queries. Our main results characterize information-theoretically minimal views that support the querying of a given CQ.

We note that our main results hold for the variant of the problem where quantification in the utility and non-disclosure definitions is considered over all instances and the one where the quantification is considered over only finite instances. There has been a long-running discussion over which quantification is to be preferred. We adopt the version with unrestricted instances as a default, following quite a number of works in this area (e.g. [22]). This is primarily for technical convenience, since it allows us to employ the chase as a technique without assuming termination, which provides more intuitive proofs in certain cases. In Section 4 we show that the analysis using shuffles can provide alternative - albeit more complex - proofs of many of our main results without relying on infinite instances. However, we note one case where we have not explored the situation when the quantification is over finite instances: Theorem 5.5 in the case of local constraints that do not have terminating chase.

We now look first at the high-level take-aways of our results, and future directions based on these. We then turn to some formal problems left open by our work.

Outlook for practice. Standard approaches to database privacy give guarantees that rely on limiting the computational resources of the attacker, or on modelling disclosure mechanisms probabilistically, with the corresponding guarantees being probabilistic. The framework in this work is radically different in that disclosure mechanisms and 
secrets are modelled deterministically. The corresponding utility and privacy guarantees are incomparable to prior work, but they are strong in many senses; e.g. the views must allow the utility query to be answered exactly on every instance. Our results in Section 3 can be considered negative, and a validation of the current approaches: If we use CQ views the only secret queries we can protect in this model are the obvious ones. The main results in Section 4 are nearly as discouraging. There we show, surprisingly, that by exploiting symmetries in the utility query we can protect some secrets by using some non-trivial views. But these are the only secrets we can preserve, and they are hardly natural. Section 5 shows that this pessimistic story line extends to the presence of local constraints.

The results in Section 6 are more encouraging. We show that in the presence of replication that non-trivial trade-offs can be achieved even for very simple utility queries, with views that are quite involved. Like any non-local constraints, replication constraints must rely on some communication mechanism between sources for their enforcement. These results can thus be interpreted is saying that if such a replication mechanism is in place, a large class of queries can be supported without revealing more than the minimal set of secrets - those that are entailed. However the results are limited to UN non-disclosure and it remains to be seen whether they can be extended to non-disclosure functions that are more interesting for practice.

Outlook for foundations of view design. We see the main significance of our results more broadly as making a contribution to understanding view design. One long-term goal would be a framework for specifying properties of views and a corresponding set of algorithms for synthesizing views that meet a given specification. Recent research [23] has indicated that determinacy can play a fundamental role in ordering views by expressiveness, and so it is natural to first gain a better grasp of this ordering. Our notion of minimal information view and our characterization of what such views look like in special cases gives new insight into determinacy. It can be seen as an - admittedly very small — step towards a framework for view synthesis.

Open questions. In this work we consider only a limited setting; e.g. CQs for the utility query and the secret query. Our hope is that the work can serve as a basis for further exploration of the trade-offs in using query-based mechanisms in a variety of settings.

Even in this restricted setting, our contribution focuses primarily on expressiveness, leaving open many questions of decidability and complexity. In particular we do not know whether the $\Sigma_{2}^{p}$ bound of Corollary 3.6 is tight. Nor do we know whether the analogous question for arbitrary views - whether there is an arbitrary d-view that is useful for a given $Q$ but UN non-disclosing for a given $p$ - is even decidable. Our results reduce this to a non-disclosure question for the shuffle views.

We have restricted to constraints that are TGDs within this paper. For our results on CQ-based views it is routine to extend to Equality-Generating Dependencies (EGDs) as well, since we rely only on some properties of the chase that hold for EGDs as well as TGDs. However, as we have shown in Example 5.4, the results on CQ-based views do not generalize to other common classes of integrity constraints. On the other hand, we believe our results on arbitrary views extend to the presence of any background knowledge. We defer the investigation of the borderline to future work. 


\section{REFERENCES}

[1] Serge Abiteboul, Richard Hull, and Victor Vianu. 1995. Foundations of Databases. Addison-Wesley.

[2] Marcelo Arenas, Pablo Barceló, and Juan L. Reutter. 2011. Query Languages for Data Exchange: Beyond Unions of Conjunctive Queries. Theory Comput. Syst. 49, 2 (2011), 489-564.

[3] Jean-François Baget, Fabien Garreau, Marie-Laure Mugnier, and Swan Rocher. 2014. Extending Acyclicity Notions for Existential Rules. In ECAI.

[4] Jean-François Baget, Marie-Laure Mugnier, Sebastian Rudolph, and Michaël Thomazo. 2011. Walking the Complexity Lines for Generalized Guarded Existential Rules. In IJCAI.

[5] Vince Bárány, Michael Benedikt, and Balder Ten Cate. 2018. Some Model Theory Of Guarded Nengation. The Journal of Symbolic Logic 83, 4 (2018), 1307-1344.

[6] Johes Bater, Gregory Elliott, Craig Eggen, Satyender Goel, Abel N. Kho, and Jennie Rogers. 2017. SMCQL: Secure Query Processing for Private Data Networks. In VLDB.

[7] Michael Benedikt, Pierre Bourhis, Louis Jachiet, and Michaël Thomazo. 2019. Reasoning about Disclosure in Data Integration in the Presence of Source Constraints. In IJCAI.

[8] Michael Benedikt, Pierre Bourhis, Balder ten Cate, and Gabriele Puppis. 2016. Querying Visible and Invisible Information. In LICS.

[9] Michael Benedikt, Bernardo Cuenca Grau, and Egor V. Kostylev. 2018. Logical foundations of information disclosure in ontology-based data integration. Artif. Intell. 262 (2018), 52-95.

[10] Michael Benedikt, Balder ten Cate, and Efi Tsamoura. 2016. Generating plans from proofs. In TODS.

[11] Piero A. Bonatti and Luigi Sauro. 2013. A Confidentiality Model for Ontologies. In ISWC.

[12] Diego Calvanese, Giuseppe De Giacomo, Maurizio Lenzerini, and Riccardo Rosati. 2012. View-based Query Answering in Description Logics: Semantics and Complexity. J. Comput. Syst. Sci. 78, 1 (2012), 26-46.

[13] David Chaum, Claude Crépeau, and Ivan Damgard. 1988. Multiparty Unconditionally Secure Protocols. In STOC.

[14] Bernardo Cuenca Grau, Ian Horrocks, Markus Krötzsch, Clemens Kupke, Despoina Magka, Boris Motik, and Zhe Wang. 2013. Acyclicity Notions for Existential Rules and Their Application to Query Answering in Ontologies. JAIR 47 (2013), 741-808.

[15] A. Deutsch, A. Nash, and J. Remmel. 2008. The Chase Revisited. In PODS.

[16] Cynthia Dwork. 2006. Differential Privacy. In ICALP.

[17] Cynthia Dwork and Aaron Roth. 2014. The Algorithmic Foundations of Differential Privacy. Found. \& Trends in Th. Comp. Sci. 9, 3\&4 (Aug. 2014), 211-407.

[18] Ronald Fagin, Phokion G. Kolaitis, Renee J. Miller, and Lucian Popa. 2005. Data Exchange: Semantics and Query Answering. Theoretical Computer Science 336, 1 (2005), 89-124.

[19] Tomasz Gogacz and Jerzy Marcinkowski. 2015. The Hunt for a Red Spider: Conjunctive Query Determinacy Is Undecidable. In LICS.

[20] Tomasz Gogacz and Jerzy Marcinkowski. 2016. Red Spider Meets a Rainworm: Conjunctive Query Finite Determinacy Is Undecidable. In PODS.

[21] Alon Y. Halevy. 2001. Answering queries using views: A survey. VLDB J. 10, 4 (2001), 270-294.

[22] David S. Johnson and Anthony C. Klug. 1984. Testing Containment of Conjunctive Queries under Functional and Inclusion Dependencies. JCSS 28, 1 (1984).

[23] Paraschos Koutris, Prasang Upadhyaya, Magdalena Balazinska, Bill Howe, and Dan Suciu. 2015. Query-Based Data Pricing. J. ACM 62, 5 (2015).

[24] Chao Li, Daniel Yang Li, Gerome Miklau, and Dan Suciu. 2017. A theory of pricing private data. Commun. ACM 60, 12 (2017), 79-86.

[25] D. Maier, A. O. Mendelzon, and Y. Sagiv. 1979. Testing implications of data dependencies. TODS 4, 4 (1979), 455-469.

[26] B. Marnette. 2009. Generalized schema-mappings: from termination to tractability. In PODS.

[27] Alan Nash and Alin Deutsch. 2007. Privacy in GLAV Information Integration. In ICDT.

[28] Alan Nash, Luc Segoufin, and Victor Vianu. 2010. Views and queries: Determinacy and rewriting. TODS 35, 3 (2010).

[29] A. Onet. 2013. The Chase Procedure and its Applications in Data Exchange. In DEIS. 1-37. 